\title{
Modified Hybrid Steepest-Descent Methods for General Systems of Variational Inequalities with Solutions to Zeros of $m$-Accretive Operators in Banach Spaces
}

\author{
Lu-Chuan Ceng ${ }^{1,2}$ and Ching-Feng Wen ${ }^{3}$ \\ ${ }^{1}$ Department of Mathematics, Shanghai Normal University, Shanghai 200234, China \\ ${ }^{2}$ Scientific Computing Key Laboratory of Shanghai Universities, Shanghai 200234, China \\ ${ }^{3}$ Center for Fundamental Science, Kaohsiung Medical University, Kaohsiung 807, Taiwan \\ Correspondence should be addressed to Ching-Feng Wen; cfwen@kmu.edu.tw
}

Received 1 July 2013; Accepted 28 July 2013

Academic Editor: Chi-Ming Chen

Copyright (C) 2013 L.-C. Ceng and C.-F. Wen. This is an open access article distributed under the Creative Commons Attribution License, which permits unrestricted use, distribution, and reproduction in any medium, provided the original work is properly cited.

\begin{abstract}
The purpose of this paper is to introduce and analyze modified hybrid steepest-descent methods for a general system of variational inequalities (GSVI), with solutions being also zeros of an $m$-accretive operator $A$ in the setting of real uniformly convex and 2 uniformly smooth Banach space X. Here the modified hybrid steepest-descent methods are based on Korpelevich's extragradient method, hybrid steepest-descent method, and viscosity approximation method. We propose and consider modified implicit and explicit hybrid steepest-descent algorithms for finding a common element of the solution set of the GSVI and the set $A^{-1}(0)$ of zeros of $A$ in $X$. Under suitable assumptions, we derive some strong convergence theorems. The results presented in this paper improve, extend, supplement, and develop the corresponding results announced in the earlier and very recent literature.
\end{abstract}

\section{Introduction}

Let $X$ be a real Banach space whose dual space is denoted by $X^{*}$. The normalized duality mapping $J: X \rightarrow 2^{X^{*}}$ is defined by

$$
J(x)=\left\{x^{*} \in X^{*}:\left\langle x, x^{*}\right\rangle=\|x\|^{2}=\left\|x^{*}\right\|^{2}\right\}, \quad \forall x \in X,
$$

where $\langle\cdot, \cdot\rangle$ denotes the generalized duality pairing. It is an immediate consequence of the Hahn-Banach theorem that $J(x)$ is nonempty for each $x \in X$. Let $C$ be a nonempty closed convex subset of $X$. A mapping $T: C \rightarrow C$ is called nonexpansive if $\|T x-T y\| \leq\|x-y\|$ for every $x, y \in C$. The set of fixed points of $T$ is denoted by $\operatorname{Fix}(T)$. We use the notation $\rightarrow$ to indicate the weak convergence and $\rightarrow$ to indicate the strong convergence. A mapping $A: C \rightarrow X$ is said to be

(i) accretive if for each $x, y \in C$ there exists $j(x-y) \in$ $J(x-y)$ such that

$$
\langle A x-A y, j(x-y)\rangle \geq 0
$$

(ii) $\alpha$-strongly accretive if for each $x, y \in C$ there exists $j(x-y) \in J(x-y)$ such that

$$
\langle A x-A y, j(x-y)\rangle \geq \alpha\|x-y\|^{2},
$$

for some $\alpha \in(0,1)$;

(iii) $\beta$-inverse strongly accretive if for each $x, y \in C$ there exists $j(x-y) \in J(x-y)$ such that

$$
\langle A x-A y, j(x-y)\rangle \geq \beta\|A x-A y\|^{2},
$$

for some $\beta>0$;

(iv) $\lambda$-strictly pseudocontractive [1] (see also [2]) if for each $x, y \in C$ there exists $j(x-y) \in J(x-y)$ such that

$$
\langle A x-A y, j(x-y)\rangle \leq\|x-y\|^{2}-\lambda\|x-y-(A x-A y)\|^{2}
$$

for some $\lambda \in(0,1)$.

It is worth emphasizing that the definition of the inverse strongly accretive mapping is based on that of the inverse 
strongly monotone mapping, which was studied by so many authors; see, for example, [3-5]. Let $U=\{x \in X:\|x\|=1\}$ denote the unite sphere of $X$. A Banach space $X$ is said to be uniformly convex if for each $\epsilon \in(0,2]$ there exists $\delta>0$ such that for all $x, y \in U$

$$
\|x-y\| \geq \epsilon \Longrightarrow \frac{\|x+y\|}{2} \leq 1-\delta .
$$

It is known that a uniformly convex Banach space is reflexive and strictly convex. A Banach space $X$ is said to be smooth if the limit

$$
\lim _{t \rightarrow 0} \frac{\|x+t y\|-\|x\|}{t}
$$

exists for all $x, y \in U$; in this case, $X$ is also said to have a Gateaux differentiable norm. Moreover, it is said to be uniformly smooth if this limit is attained uniformly for $x, y \in$ $U$; in this case, $X$ is also said to have a uniformly Frechet differentiable norm. The norm of $X$ is said to be the Frechet differential if for each $x \in U$ this limit is attained uniformly for $y \in U$. In the meantime, we define a function $\rho$ : $[0, \infty) \rightarrow[0, \infty)$ called the modulus of smoothness of $X$ as follows:

$$
\begin{array}{r}
\rho(\tau)=\sup \left\{\frac{1}{2}(\|x+y\|+\|x-y\|)-1:\right. \\
x, y \in X,\|x\|=1,\|y\|=\tau\} .
\end{array}
$$

It is known that $X$ is uniformly smooth if and only if $\lim _{\tau \rightarrow 0} \rho(\tau) / \tau=0$. Let $q$ be a fixed real number with $1<q \leq$ 2. Then a Banach space $X$ is said to be $q$-uniformly smooth if there exists a constant $c>0$ such that $\rho(\tau) \leq c \tau^{q}$ for all $\tau>0$. As pointed out in [6], no Banach space is $q$-uniformly smooth for $q>2$. In addition, it is also known that $J$ is single-valued if and only if $X$ is smooth, whereas if $X$ is uniformly smooth, then the mapping $J$ is norm-to-norm uniformly continuous on bounded subsets of $X$.

Let $A$ be an operator with domain $D(A)$ and range $R(A)$ in $X$ is said to be accretive if for each $x_{i} \in D(A)$ and $y_{i} \in$ $A x_{i}(i=1,2)$ there exists $j\left(x_{2}-x_{1}\right) \in J\left(x_{2}-x_{1}\right)$ such that

$$
\left\langle y_{2}-y_{1}, j\left(x_{2}-x_{1}\right)\right\rangle \geq 0 \text {. }
$$

An accretive operator $A$ is said to be $m$-accretive if $R(I+$ $\lambda A)=X$ for all $\lambda>0$. Denote by $J_{r}$ the resolvent of $A$; that is, for each $r>0$,

$$
J_{r}=(I+r A)^{-1}
$$

It is known that $J_{r}$ is a nonexpansive mapping from $X$ to $C:=\overline{D(A)}$ which will be assumed convex. In 2008, Chen and Zhu [7] derived the following strong convergence theorems for viscosity approximation methods for accretive operators in a uniformly smooth Banach space $X$.

Theorem CZ1. Let $X$ be a uniformly smooth Banach space. Suppose that $A$ is an m-accretive operator in $X$ such that
$C=\overline{D(A)}$ is convex and $f: C \rightarrow C$ is a fixed contractive map. For each $t \in(0,1),\left\{x_{t, n}\right\}$ is defined by

$$
x_{t, n}=t f\left(x_{t, n}\right)+(1-t) J_{r_{n}} x_{t, n} .
$$

Then as $t \rightarrow 0,\left\{x_{t, n}\right\}$ converges strongly to a zero of $A$.

Theorem CZ2. Let $X$ be a uniformly smooth Banach space. Suppose that $A$ is an $m$-accretive operator in $X$ such that $C=$ $\overline{D(A)}$ is convex and $f: C \rightarrow C$ is a fixed contractive map. The sequence $\left\{x_{n}\right\}$ is defined by

$$
x_{n+1}=\alpha_{n} f\left(x_{n}\right)+\left(1-\alpha_{n}\right) J_{r_{n}} x_{n}, \quad \forall n \geq 0,
$$

where $\left\{\alpha_{n}\right\}$ and $\left\{r_{n}\right\}$ satisfy the following conditions:

$$
\begin{aligned}
& \text { (i) } \alpha_{n} \rightarrow 0, \sum_{n=0}^{\infty} \alpha_{n}=\infty \text {, and } \sum_{n=0}^{\infty}\left|\alpha_{n+1}-\alpha_{n}\right|<\infty \text {, } \\
& \text { (ii) } r_{n} \geq \varepsilon \text { for all } n \text { and } \sum_{n=0}^{\infty}\left|r_{n+1}-r_{n}\right|<\infty \text {. }
\end{aligned}
$$

Then as $n \rightarrow \infty,\left\{x_{n}\right\}$ converges strongly to a zero of $A$.

In the meantime, Ceng et al. [8] derived some strong convergence theorems of composite iterative schemes for zeros of $m$-accretive operators in uniformly smooth Banach spaces. Furthermore, motivated by strong convergence results for hybrid steepest-descent methods in $[9,10]$, Ceng et al. [11] established some strong convergence theorems for hybrid steepest-descent methods for nonexpansive and $m$-accretive operators in a uniformly smooth Banach space $X$. Subsequently, Ceng et al. [12] introduced hybrid viscosity approximation method for finding zeros of $m$-accretive operators, which combine viscosity approximation method with hybrid steepest-descent method, and obtained the following strong convergence theorems.

Theorem CASY1. Let $X$ be a uniformly smooth Banach space, let $A$ be an $m$-accretive operator in $X$ with $A^{-1}(0) \neq \emptyset$, and let $f: X \rightarrow C(=\overline{D(A)})$ be a contractive map. Assume that $F: X \rightarrow X$ is $\delta$-strongly accretive and $\lambda$-strictly pseudocontractive with $\delta+\lambda>1$. For each $t \in(0,1)$ and each integer $n \geq 0$, let $\left\{x_{t, n}\right\}$ be defined by

$$
x_{t, n}=t f\left(x_{t, n}\right)+(1-t)\left[J_{r_{n}} x_{t, n}-\theta_{t} F\left(x_{t, n}\right)\right]
$$

where $\left\{r_{n}\right\} \subset[\varepsilon, \infty)$ for some $\varepsilon>0$ and $\left\{\theta_{t}: t \in(0,1)\right\} \subset[0,1)$ with $\lim _{t \rightarrow 0}\left(\theta_{t} / t\right)=0$. Then as $t \rightarrow 0,\left\{x_{t, n}\right\}$ converges strongly to a zero $p$ of $A$, which is a unique solution of the variational inequality problem (VIP)

$$
\langle(\mathrm{I}-f) p, J(p-u)\rangle \leq 0, \quad \forall u \in A^{-1}(0) .
$$

Theorem CASY2. Let $X$ be a uniformly smooth Banach space, let $A$ be an $m$-accretive operator in $X$ with $A^{-1}(0) \neq \emptyset$, and let $f: X \rightarrow C(=\overline{D(A)})$ be a contractive map. Assume that $F: X \rightarrow X$ is $\delta$-strongly accretive and $\lambda$ strictly pseudocontractive with $\delta+\lambda>1$. Given sequences $\left\{\lambda_{n}\right\}_{n=0}^{\infty},\left\{\mu_{n}\right\}_{n=0}^{\infty}$ in $[0,1],\left\{\alpha_{n}\right\}_{n=0}^{\infty},\left\{\beta_{n}\right\}_{n=0}^{\infty}$ in $(0,1]$, and $\left\{r_{n}\right\}_{n=0}^{\infty}$ 
in $[\varepsilon, \infty)$ for some $\varepsilon>0$, suppose that there hold the following conditions:

(i) $\lim _{n \rightarrow \infty} \beta_{n}=0$ and $\sum_{n=0}^{\infty} \beta_{n}=\infty$,

(ii) $\lim _{n \rightarrow \infty}\left(\lambda_{n} \mu_{n}\right) / \beta_{n}=0$,

(iii) $\left\{\alpha_{n}\right\} \subset[a, b]$ for some $a, b \in(0,1)$,

(iv) $\sum_{n=0}^{\infty}\left|\alpha_{n+1}-\alpha_{n}\right|<\infty, \sum_{n=0}^{\infty}\left|\beta_{n+1}-\beta_{n}\right|<\infty$, $\sum_{n=0}^{\infty}\left|\lambda_{n+1}-\lambda_{n}\right|<\infty, \sum_{n=0}^{\infty}\left|\mu_{n+1}-\mu_{n}\right|<\infty$ and

Then for any given point $x_{0} \in X$, the sequence $\left\{x_{n}\right\}$ generated by

$$
\begin{aligned}
& y_{n}=\alpha_{n} x_{n}+\left(1-\alpha_{n}\right) J_{r_{n}} x_{n}, \\
& x_{n+1}=\beta_{n} f\left(x_{n}\right)+\left(1-\beta_{n}\right)\left[J_{r_{n}} y_{n}-\lambda_{n} \mu_{n} F\left(J_{r_{n}} y_{n}\right)\right], \\
& \forall n \geq 0,
\end{aligned}
$$

converges strongly to a zero $p$ of $A$, which is a unique solution of the VIP as above.

On the other hand, $\mathrm{Cai}$ and $\mathrm{Bu}$ [13] considered the following general system of variational inequalities (GSVI) in a real smooth Banach space $X$, which involves finding $\left(x^{*}, y^{*}\right) \in C \times C$ such that

$$
\begin{aligned}
& \left\langle\mu_{1} B_{1} y^{*}+x^{*}-y^{*}, J\left(x-x^{*}\right)\right\rangle \geq 0, \quad \forall x \in C, \\
& \left\langle\mu_{2} B_{2} x^{*}+y^{*}-x^{*}, J\left(x-y^{*}\right)\right\rangle \geq 0, \quad \forall x \in C,
\end{aligned}
$$

where $C$ is a nonempty, closed and convex subset of $X, B_{1}, B_{2}: C \rightarrow X$ are two nonlinear mappings, and $\mu_{1}$ and $\mu_{2}$ are two positive constants. Here the set of solutions of GSVI (16) is denoted by GSVI $\left(C, B_{1}, B_{2}\right)$. In particular, if $X=H$, a real Hilbert space, then GSVI (16) reduces to the following GSVI of finding $\left(x^{*}, y^{*}\right) \in C \times C$ such that

$$
\begin{array}{ll}
\left\langle\mu_{1} B_{1} y^{*}+x^{*}-y^{*}, x-x^{*}\right\rangle \geq 0, & \forall x \in C, \\
\left\langle\mu_{2} B_{2} x^{*}+y^{*}-x^{*}, x-y^{*}\right\rangle \geq 0, & \forall x \in C,
\end{array}
$$

where $\mu_{1}$ and $\mu_{2}$ are two positive constants. The set of solutions of problem (17) is still denoted by GSVI $\left(C, B_{1}, B_{2}\right)$. In particular, if $B_{1}=B_{2}=A$, then problem (17) reduces to the new system of variational inequalities (NSVI), introduced and studied by Verma [14]. Further, if $x^{*}=y^{*}$ additionally, then the NSVI reduces to the classical variational inequality problem (VIP) of finding $x^{*} \in C$ such that

$$
\left\langle A x^{*}, x-x^{*}\right\rangle \geq 0, \quad \forall x \in C .
$$

The solution set of the VIP (18) is denoted by $\operatorname{VI}(C, A)$. Variational inequality theory has been studied quite extensively and has emerged as an important tool in the study of a wide class of obstacle, unilateral, free, moving, equilibrium problems. It is now well known that the variational inequalities are equivalent to the fixed point problems, the origin of which can be traced back to Lions and Stampacchia [15]. This alternative formulation has been used to suggest and analyze projection iterative method for solving variational inequalities under the condition that the involved operator must be strongly monotone and Lipschitz continuous.

Recently, Ceng et al. [16] transformed problem (17) into a fixed point problem in the following way.

Lemma 1 (see [16]). For given $\bar{x}, \bar{y} \in C,(\bar{x}, \bar{y})$ is a solution of problem (17) if and only if $\bar{x}$ is a fixed point of the mapping $G: C \rightarrow C$ defined by

$$
\begin{array}{r}
G(x)=P_{C}\left[P_{C}\left(x-\mu_{2} B_{2} x\right)-\mu_{1} B_{1} P_{C}\left(x-\mu_{2} B_{2} x\right)\right], \\
\forall x \in C,
\end{array}
$$

where $\bar{y}=P_{C}\left(\bar{x}-\mu_{2} B_{2} \bar{x}\right)$ and $P_{C}$ is the the projection of $H$ onto C.

In particular, if the mapping $B_{i}: C \rightarrow H$ is $\beta_{i^{-}}$ inverse strongly monotone for $i=1,2$, then the mapping $G$ is nonexpansive provided $\mu_{i} \in\left(0,2 \beta_{i}\right)$ for $i=1,2$.

In 1976, Korpelevič [17] proposed an iterative algorithm for solving the VIP (18) in Euclidean space $\mathbf{R}^{n}$ :

$$
\begin{array}{r}
y_{n}=P_{C}\left(x_{n}-\tau A x_{n}\right), \quad x_{n+1}=P_{C}\left(x_{n}-\tau A y_{n}\right), \\
\forall n \geq 0,
\end{array}
$$

with $\tau>0$ being a given number, which is known as the extragradient method (see also [18]). The literature on the VIP is vast, and Korpelevich's extragradient method has received great attention given by many authors, who improved it in various ways; see, for example, [3, 13, 19-32] and references therein, to name but a few.

In particular, whenever $X$ is still a real smooth Banach space, $B_{1}=B_{2}=A$, and $x^{*}=y^{*}$, then GSVI (16) reduces to the variational inequality problem (VIP) of finding $x^{*} \in C$ such that

$$
\left\langle A x^{*}, J\left(x-x^{*}\right)\right\rangle \geq 0, \quad \forall x \in C,
$$

which was considered by Aoyama et al. [33]. Note that VIP (21) is connected with the fixed point problem for nonlinear mapping (see, e.g., [34]), the problem of finding a zero point of a nonlinear operator (see, e.g., [35]), and so on. It is clear that VIP (21) extends VIP (18) from Hilbert spaces to Banach spaces.

In order to find a solution of VIP (21), Aoyama et al. [33] introduced the following iterative scheme for an accretive operator $A$ :

$$
x_{n+1}=\alpha_{n} x_{n}+\left(1-\alpha_{n}\right) \Pi_{C}\left(x_{n}-\lambda_{n} A x_{n}\right), \quad \forall n \geq 1,
$$

where $\Pi_{C}$ is a sunny nonexpansive retraction from $X$ onto $C$. Then they proved a weak convergence theorem.

Beyond doubt, it is an interesting and valuable problem of constructing some algorithms with strong convergence for solving GSVI (16) which contains VIP (21) as a special case. Very recently, Cai and $\mathrm{Bu}$ [13] constructed an iterative algorithm for solving GSVI (16) and a common fixed point problem of an infinite family of nonexpansive mappings in a uniformly convex and 2-uniformly smooth Banach space. They proved the strong convergence of the proposed algorithm by virtue of the following inequality in a 2-uniformly smooth Banach space $X$. 
Lemma 2 (see [36]). Let $X$ be a 2-uniformly smooth Banach space. Then

$$
\|x+y\|^{2} \leq\|x\|^{2}+2\langle y, J(x)\rangle+2\|\kappa y\|^{2}, \quad \forall x, y \in X,
$$

where $\kappa$ is the 2-uniformly smooth constant of $X$ and $J$ is the normalized duality mapping from $X$ into $X^{*}$.

Define the mapping $G: C \rightarrow C$ as follows:

$$
G(x):=\Pi_{C}\left(I-\mu_{1} B_{1}\right) \Pi_{C}\left(I-\mu_{2} B_{2}\right) x, \quad \forall x \in C .
$$

The fixed point set of $G$ is denoted by $\Omega$. Then their strong convergence theorem on the proposed method is stated as follows.

Theorem CB (see [13, Theorem 3.1]). Let $C$ be a nonempty closed convex subset of a uniformly convex and 2-uniformly smooth Banach space $X$. Let $\Pi_{C}$ be a sunny nonexpansive retraction from $X$ onto $C$. Let the mapping $B_{i}: C \rightarrow X$ be $\alpha_{i}$-inverse strongly accretive with $0<\mu_{i}<\alpha_{i} / \kappa^{2}$ for $i=1,2$. Let $f$ be a contraction of $C$ into itself with coefficient $\rho \in(0,1)$. Let $\left\{S_{n}\right\}_{n=1}^{\infty}$ be an infinite family of nonexpansive mappings of $C$ into itself such that $\Delta=\bigcap_{i=1}^{\infty} \operatorname{Fix}\left(S_{i}\right) \cap \Omega \neq \emptyset$, where $\Omega$ is the fixed point set of the mapping $G$ defined by (24). For arbitrarily given $x_{1} \in C$, let $\left\{x_{n}\right\}$ be the sequence generated by

$$
\begin{array}{r}
x_{n+1}=\beta_{n} x_{n}+\left(1-\beta_{n}\right) S_{n} y_{n}, \\
y_{n}=\alpha_{n} f\left(x_{n}\right)+\left(1-\alpha_{n}\right) z_{n}, \\
z_{n}=\Pi_{C}\left(u_{n}-\mu_{1} B_{1} u_{n}\right), \\
u_{n}=\Pi_{C}\left(x_{n}-\mu_{2} B_{2} x_{n}\right), \\
\forall n \geq 1 .
\end{array}
$$

Suppose that $\left\{\alpha_{n}\right\}$ and $\left\{\beta_{n}\right\}$ are two sequences in $(0,1)$ satisfying the following conditions:

(i) $\lim _{n \rightarrow \infty} \alpha_{n}=0$ and $\sum_{n=1}^{\infty} \alpha_{n}=\infty$,

(ii) $0<\liminf _{n \rightarrow \infty} \beta_{n} \leq \lim \sup _{n \rightarrow \infty} \beta_{n}<1$.

Assume that $\sum_{n=1}^{\infty} \sup _{x \in D}\left\|S_{n+1} x-S_{n} x\right\|<\infty$ for any bounded subset $D$ of $C$, let $S$ be a mapping of $C$ into $X$ defined by $S x=\lim _{n \rightarrow \infty} S_{n} x$ for all $x \in C$, and suppose that $\operatorname{Fix}(S)=$ $\bigcap_{n=1}^{\infty} \operatorname{Fix}\left(S_{n}\right)$. Then $\left\{x_{n}\right\}$ converges strongly to $p \in \Delta$, which solves the VIP:

$$
\langle(I-f) p, J(p-u)\rangle \leq 0, \quad \forall u \in \Delta .
$$

It is easy to see that the iterative scheme in Theorem $C B$ is essentially equivalent to the following two-step iterative scheme:

$$
\begin{array}{r}
y_{n}=\alpha_{n} f\left(x_{n}\right)+\left(1-\alpha_{n}\right) G x_{n}, \\
x_{n+1}=\beta_{n} x_{n}+\left(1-\beta_{n}\right) S_{n} y_{n}, \\
\forall n \geq 1 .
\end{array}
$$

For the convenience of implementing the argument techniques in [16], the authors [13] have used the following inequality in a real smooth and uniform convex Banach space $X$.
Proposition 3 (see [37]). Let $X$ be a real smooth and uniform convex Banach space and let $r>0$. Then there exists a strictly increasing, continuous and convex function $g:[0,2 r] \rightarrow \mathbf{R}$, $g(0)=0$, such that

$$
g(\|x-y\|) \leq\|x\|^{2}-2\langle x, J(y)\rangle+\|y\|^{2}, \quad \forall x, y \in B_{r},
$$

where $B_{r}=\{x \in X:\|x\| \leq r\}$.

Let $C$ be a nonempty closed convex subset of a real uniformly convex and 2-uniformly smooth Banach space $X$. Let $\Pi_{C}$ be a sunny nonexpansive retraction from $X$ onto $C$ and let $f: C \rightarrow C$ be a contraction with coefficient $\rho \in(0,1)$. Motivated and inspired by the research going on in this area, we introduce and analyze modified hybrid steepest-descent methods for the GSVI (16), with solutions being also zeros of an $m$-accretive operator $A$ in $X$. Here the modified hybrid steepest-descent methods are based on Korpelevich's extragradient method, hybrid steepest-descent method, and viscosity approximation method. We propose and consider modified implicit and explicit hybrid steepestdescent algorithms for finding a common element of the solution set of the GSVI (16) and the set $A^{-1}(0)$ of zeros of $A$ in $X$. Under suitable assumptions, we derive some strong convergence theorems. The results presented in this paper improve, extend, supplement, and develop the corresponding results announced in the earlier and very recent literature $[12,13,16,32]$.

\section{Preliminaries}

We list some lemmas that will be used in the sequel. Lemma 4 can be found in [38]. Lemma 5 is an immediate consequence of the subdifferential inequality of the function $(1 / 2)\|\cdot\|^{2}$.

Lemma 4. Let $\left\{a_{n}\right\}$ be a sequence of nonnegative real numbers such that

$$
a_{n+1} \leq\left(1-b_{n}\right) a_{n}+b_{n} c_{n}, \quad \forall n \geq 0
$$

where $\left\{b_{n}\right\}$ and $\left\{c_{n}\right\}$ are sequences of real numbers satisfying the following conditions:

(i) $\left\{b_{n}\right\} \subset[0,1]$ and $\sum_{n=0}^{\infty} b_{n}=\infty$,

(ii) either $\lim \sup _{n \rightarrow \infty} c_{n} \leq 0$ or $\sum_{n=0}^{\infty}\left|b_{n} c_{n}\right|<\infty$.

Then, $\lim _{n \rightarrow \infty} a_{n}=0$.

Lemma 5. In a real smooth Banach space $X$, there holds the inequality

$$
\begin{aligned}
\|x\|^{2}+2\langle y, J(x)\rangle & \leq\|x+y\|^{2} \\
& \leq\|x\|^{2}+2\langle y, J(x+y)\rangle, \quad \forall x, y \in X,
\end{aligned}
$$

where $J: X \rightarrow X^{*}$ is the normalized duality mapping. 
Let $D$ be a subset of $C$ and let $\Pi$ be a mapping of $C$ into $D$. Then $\Pi$ is said to be sunny if

$$
\Pi[\Pi(x)+t(x-\Pi(x))]=\Pi(x),
$$

whenever $\Pi(x)+t(x-\Pi(x)) \in C$ for $x \in C$ and $t \geq 0$. A mapping $\Pi$ of $C$ into itself is called a retraction if $\Pi^{2}=\Pi$. If a mapping $\Pi$ of $C$ into itself is a retraction, then $\Pi(z)=z$ for every $z \in R(\Pi)$, where $R(\Pi)$ is the range of $\Pi$. A subset $D$ of $C$ is called a sunny nonexpansive retract of $C$ if there exists a sunny nonexpansive retraction from $C$ onto $D$. The following lemma concerns the sunny nonexpansive retraction.

Lemma 6 (see [39]). Let $C$ be a nonempty closed convex subset of a real smooth Banach space $X$. Let $D$ be a nonempty subset of $C$. Let $\Pi$ be a retraction of $C$ onto $D$. Then the following are equivalent:

(i) $\Pi$ is sunny and nonexpansive,

(ii) $\|\Pi(x)-\Pi(y)\|^{2} \leq\langle x-y, J(\Pi(x)-\Pi(y))\rangle, \forall x, y \in C$,

(iii) $\langle x-\Pi(x), J(y-\Pi(x))\rangle \leq 0, \forall x \in C, y \in D$.

It is well known that if $X=H$, a Hilbert space, then a sunny nonexpansive retraction $\Pi_{C}$ is coincident with the metric projection from $X$ onto $C$; that is, $\Pi_{C}=P_{C}$. If $C$ is a nonempty closed convex subset of a strictly convex and uniformly smooth Banach space $X$ and if $T: C \rightarrow C$ is a nonexpansive mapping with the fixed point set $\operatorname{Fix}(T) \neq \emptyset$, then the set $\operatorname{Fix}(T)$ is a sunny nonexpansive retract of $C$. The following lemma follows easily from Lemma 6.

Lemma 7. Let $C$ be a nonempty closed convex subset of a smooth Banach space $X$. Let $\Pi_{C}$ be a sunny nonexpansive retraction from $X$ onto $C$ and let $B_{1}, B_{2}: C \rightarrow X$ be nonlinear mappings. For given $x^{*}, y^{*} \in C,\left(x^{*}, y^{*}\right)$ is a solution of GSVI (16) if and only if $x^{*}=\Pi_{C}\left(y^{*}-\mu_{1} B_{1} y^{*}\right)$, where $y^{*}=$ $\Pi_{C}\left(x^{*}-\mu_{2} B_{2} x^{*}\right)$.

In terms of Lemma 7, we observe that

$$
x^{*}=\Pi_{C}\left[\Pi_{C}\left(x^{*}-\mu_{2} B_{2} x^{*}\right)-\mu_{1} B_{1} \Pi_{C}\left(x^{*}-\mu_{2} B_{2} x^{*}\right)\right],
$$

which implies that $x^{*}$ is a fixed point of the mapping $G$. Throughout this paper, the set of fixed points of the mapping $G$ is denoted by $\Omega$.

Lemma 8 is the resolvent identity which can be found in [40], and Lemma 9 can be found in [41].

Lemma 8. For $\lambda, \mu>0$, there holds the identity

$$
J_{\lambda} x=J_{\mu}\left(\frac{\mu}{\lambda} x+\left(1-\frac{\mu}{\lambda}\right) J_{\lambda} x\right), \quad \forall x \in X .
$$

Lemma 9. Assume that $c_{2} \geq c_{1}>0$. Then $\left\|J_{c_{1}} x-x\right\| \leq 2 \| J_{c_{2}} x-$ $x \|$ for all $x \in X$.

Lemma 10 (see [42]). Let $C$ be a nonempty closed convex subset of a strictly convex Banach space X. Let $\left\{T_{n}\right\}_{n=0}^{\infty}$ be a sequence of nonexpansive mappings on $C$. Suppose that $\bigcap_{n=0}^{\infty} \operatorname{Fix}\left(T_{n}\right)$ is nonempty. Let $\left\{\lambda_{n}\right\}$ be a sequence of positive numbers with $\sum_{n=0}^{\infty} \lambda_{n}=1$. Then a mapping $S$ on $C$ defined by $S x=\sum_{n=0}^{\infty} \lambda_{n} T_{n} x$ for $x \in C$ is defined well, nonexpansive, and $\operatorname{Fix}(S)=\bigcap_{n=0}^{\infty} \operatorname{Fix}\left(T_{n}\right)$ holds.

Let $\mu$ be a mean if $\mu$ is a continuous linear functional on $l^{\infty}$ satisfying $\|\mu\|=1=\mu(1)$. Then we know that $\mu$ is a mean on $\mathbf{N}$ if and only if

$$
\inf \left\{a_{n}: n \in \mathbf{N}\right\} \leq \mu(a) \leq \sup \left\{a_{n}: n \in \mathbf{N}\right\}
$$

for every $a=\left(a_{1}, a_{2}, \ldots\right) \in l^{\infty}$. According to time and circumstances, we use $\mu_{n}\left(a_{n}\right)$ instead of $\mu(a)$. A mean $\mu$ on $\mathbf{N}$ is called a Banach limit if and only if

$$
\mu_{n}\left(a_{n}\right)=\mu_{n}\left(a_{n+1}\right)
$$

for every $a=\left(a_{1}, a_{2}, \ldots\right) \in l^{\infty}$. We know that if $\mu$ is a Banach limit, then

$$
\liminf _{n \rightarrow \infty} a_{n} \leq \mu_{n}\left(a_{n}\right) \leq \limsup _{n \rightarrow \infty} a_{n}
$$

for every $a=\left(a_{1}, a_{2}, \ldots\right) \in l^{\infty}$. So if $a=\left(a_{1}, a_{2}, \ldots\right), b=$ $\left(b_{1}, b_{2}, \ldots\right) \in l^{\infty}$, and $a_{n} \rightarrow c$ (resp., $a_{n}-b_{n} \rightarrow 0$ ), as $n \rightarrow \infty$, we have

$$
\mu_{n}\left(a_{n}\right)=\mu(a)=c \quad\left(\text { resp., } \mu_{n}\left(a_{n}\right)=\mu_{n}\left(b_{n}\right)\right) .
$$

Further, it is well known that there holds the following result.

Lemma 11 (see [43]). Let $C$ be a nonempty closed convex subset of a uniformly smooth Banach space $X$. Let $\left\{x_{n}\right\}$ be a bounded sequence of $X$, let $\mu$ be a mean on $\mathbf{N}$, and let $z \in C$. Then

$$
\mu_{n}\left\|x_{n}-z\right\|^{2}=\min _{y \in C} \mu_{n}\left\|x_{n}-y\right\|^{2}
$$

if and only if

$$
\mu_{n}\left\langle y-z, J\left(x_{n}-z\right)\right\rangle \leq 0, \quad \forall y \in C,
$$

where $J$ is the normalized duality mapping of $X$.

Let $C$ be a nonempty closed convex subset of a Banach space $X$ and let $T: C \rightarrow C$ be a nonexpansive mapping with $\operatorname{Fix}(T) \neq \emptyset$. Let $\Xi_{C}$ be the set of all contractive self-mappings on $C$. For $t \in(0,1)$ and $f \in \Xi_{C}$, let $x_{t} \in C$ be the unique fixed point of the contraction $x \mapsto t f(x)+(1-t) T x$ on $C$; that is,

$$
x_{t}=t f\left(x_{t}\right)+(1-t) T x_{t} .
$$

Lemma 12 (see [34]). Let $X$ be a uniformly smooth Banach space. Let $C$ be a nonempty closed convex subset of $X$, let $T$ : $C \rightarrow C$ be a nonexpansive mapping with $\operatorname{Fix}(T) \neq \emptyset$, and let $f \in \Xi_{C}$. Then the net $\left\{x_{t}\right\}$ defined by $x_{t}=t f\left(x_{t}\right)+(1-t) T x_{t}$ converges strongly to a point in $\operatorname{Fix}(T)$. If one defines a mapping $Q: \Xi_{C} \rightarrow \operatorname{Fix}(T)$ by $Q(f):=s-\lim _{t \rightarrow 0} x_{t}$, for all $f \in \Xi_{C}$, then $Q(f)$ solves the VIP:

$$
\begin{array}{r}
\langle(I-f) Q(f), J(Q(f)-u)\rangle \leq 0, \\
\forall f \in \Xi_{C}, u \in \operatorname{Fix}(T) .
\end{array}
$$


The following proposition will be used frequently throughout this paper.

Proposition 13 (see [11]). Let $X$ be a real smooth Banach space and let $F: X \rightarrow X$ be a mapping.

(i) If $F$ is $\lambda$-strictly pseudocontractive, then $F$ is Lipschitzian with constant $1+1 / \lambda$.

(ii) If $F$ is $\delta$-strongly accretive and $\lambda$-strictly pseudocontractive with $\delta+\lambda>1$, then $I-F$ is contractive with constant $\sqrt{(1-\delta) / \lambda}$.

(iii) If $F$ is $\delta$-strongly accretive and $\lambda$-strictly pseudocontractive with $\delta+\lambda>1$, then, for any fixed number $\tau \in(0,1), I-\tau F$ is contractive with constant $1-\tau(1-$ $\sqrt{(1-\delta) / \lambda})$.

\section{Main Results}

In this section, we introduce our modified hybrid steepestdescent schemes and show the strong convergence theorems. We will need the following useful lemmas in the sequel.

Lemma 14 (see [13, Lemma 2.8]). Let $C$ be a nonempty closed convex subset of a real 2-uniformly smooth Banach space $X$. Let the mapping $B_{i}: C \rightarrow X$ be $\alpha_{i}$-inverse strongly accretive. Then, one has

$$
\begin{aligned}
& \left\|\left(I-\mu_{i} B_{i}\right) x-\left(I-\mu_{i} B_{i}\right) y\right\|^{2} \\
& \quad \leq\|x-y\|^{2}+2 \mu_{i}\left(\mu_{i} \kappa^{2}-\alpha_{i}\right)\left\|B_{i} x-B_{i} y\right\|^{2}, \quad \forall x, y \in C,
\end{aligned}
$$

for $i=1,2$, where $\mu_{i}>0$. In particular, if $0<\mu_{i} \leq \alpha_{i} / \kappa^{2}$, then $I-\mu_{i} B_{i}$ is nonexpansive for $i=1,2$.

Lemma 15 (see [13, Lemma 2.9]). Let $C$ be a nonempty closed convex subset of a real 2-uniformly smooth Banach space $X$. Let $\Pi_{C}$ be a sunny nonexpansive retraction from $X$ onto $C$. Let the mapping $B_{i}: C \rightarrow X$ be $\alpha_{i}$-inverse strongly accretive for $i=1,2$. Let $G: C \rightarrow C$ be the mapping defined by

$$
\begin{array}{r}
G x=\Pi_{C}\left[\Pi_{C}\left(x-\mu_{2} B_{2} x\right)-\mu_{1} B_{1} \Pi_{C}\left(x-\mu_{2} B_{2} x\right)\right], \\
\forall x \in C .
\end{array}
$$

If $0<\mu_{i} \leq \alpha_{i} / \kappa^{2}$ for $i=1,2$, then $G: C \rightarrow C$ is nonexpansive.

Let $X$ be a real smooth Banach space and let $A$ be an $m$-accretive operator in $X$ such that $C=\overline{D(A)}$ is convex. Let $\Pi_{C}$ be a sunny nonexpansive retraction from $X$ onto $C$. Let $F: X \rightarrow X$ be $\delta$-strongly accretive and $\lambda$-strictly pseudocontractive with $\delta+\lambda>1$. Let the mapping $B_{i}$ : $C \rightarrow X$ be $\alpha_{i}$-inverse strongly accretive for $i=1,2$ and let $f: X \rightarrow X$ be a contractive map with coefficient $\rho \in(0,1)$. In this section, we will consider the problem of finding a point $p \in \Delta=A^{-1}(0) \cap \Omega(\neq \emptyset)$, which is a unique solution of the VIP:

$$
\langle(I-f) p, J(p-u)\rangle \leq 0, \quad \forall u \in \Delta
$$

where $\Omega$ is the fixed point set of the mapping $G=\Pi_{C}(I-$ $\left.\mu_{1} B_{1}\right) \Pi_{C}\left(I-\mu_{2} B_{2}\right)$ with $0<\mu_{i} \leq \alpha_{i} / \kappa^{2}$ for $i=1,2$. For each $t \in(0,1)$ and each integer $n \geq 0$, we choose a number $\theta_{t} \in[0,1)$ arbitrarily and then consider the following mapping $\Gamma_{t, n}: X \rightarrow X$ defined as

$$
\Gamma_{t, n} x=t f(x)+(1-t)\left[G\left(J_{r_{n}} x\right)-\theta_{t} F G\left(J_{r_{n}} x\right)\right], \quad \forall x \in X .
$$

Then, $\Gamma_{t, n}: X \rightarrow X$ is a contractive map. Indeed, utilizing Proposition 13(iii) and Lemma 15, we have for all $x, y \in X$

$$
\begin{aligned}
\| & \Gamma_{t, n} x-\Gamma_{t, n} y \| \\
= & \| t f(x)+(1-t)\left[G\left(J_{r_{n}} x\right)-\theta_{t} F G\left(J_{r_{n}} x\right)\right] \\
& \quad-t f(y)-(1-t)\left[G\left(J_{r_{n}} y\right)-\theta_{t} F G\left(J_{r_{n}} y\right)\right] \| \\
\leq & t\|f(x)-f(y)\| \\
& +(1-t)\left\|\left(I-\theta_{t} F\right) G\left(J_{r_{n}} x\right)-\left(I-\theta_{t} F\right) G\left(J_{r_{n}} y\right)\right\| \\
\leq & t \rho\|x-y\|+(1-t)\left(1-\theta_{t}\left(1-\sqrt{\frac{1-\delta}{\lambda}}\right)\right) \\
& \times\left\|G\left(J_{r_{n}} x\right)-G\left(J_{r_{n}} y\right)\right\| \\
\leq & t \rho\|x-y\|+(1-t)\left(1-\theta_{\mathrm{t}}\left(1-\sqrt{\frac{1-\delta}{\lambda}}\right)\right) \\
& \quad \times\left\|J_{r_{n}} x-J_{r_{n}} y\right\| \\
\leq & t \rho\|x-y\|+(1-t)\left(1-\theta_{t}\left(1-\sqrt{\frac{1-\delta}{\lambda}}\right)\right)\|x-y\| \\
= & (1-(1-\rho) t)\|x-y\|, \\
\leq & t
\end{aligned}
$$

and hence $\Gamma_{t, n}: X \rightarrow X$ is contractive due to $(1-\rho) t \in(0,1)$. By Banach's Contraction Mapping Principle, there exists a unique fixed point $x_{t, n}$ of $\Gamma_{t, n}$ in $X$; that is,

$$
x_{t, n}=t f\left(x_{t, n}\right)+(1-t)\left[G\left(J_{r_{n}} x_{t, n}\right)-\theta_{t} F G\left(J_{r_{n}} x_{t, n}\right)\right] \text {. }
$$

Theorem 16. Let $X$ be a uniformly convex and 2-uniformly smooth Banach space and let $A$ be an $m$-accretive operator in $X$ such that $C=\overline{D(A)}$ is convex. Let $\Pi_{C}$ be a sunny nonexpansive retraction from $X$ onto $C$. Let the mapping $B_{i}: C \rightarrow X$ be $\alpha_{i^{-}}$ inverse strongly accretive for $i=1,2$, let $f: X \rightarrow C$ be a contractive map with coefficient $\rho \in(0,1)$, and let $F: X \rightarrow$ $X$ be $\delta$-strongly accretive and $\lambda$-strictly pseudocontractive with $\delta+\lambda>1$. Assume that $\Delta=A^{-1}(0) \cap \Omega \neq \emptyset$, where $\Omega$ is the fixed point set of the mapping $G=\Pi_{C}\left(I-\mu_{1} B_{1}\right) \Pi_{C}(I-$ $\left.\mu_{2} B_{2}\right)$ with $0<\mu_{i}<\alpha_{i} / \kappa^{2}$ for $i=1$, 2. For each $t \in(0,1)$ 
and each integer $n \geq 0$, let $\left\{x_{t, n}\right\}$ be defined by (47), where $\left\{r_{n}\right\} \subset[\varepsilon, \infty)$ for some $\varepsilon>0$ and $\left\{\theta_{t}: t \in(0,1)\right\} \subset[0,1)$ with $\lim _{t \rightarrow 0}\left(\theta_{t} / t\right)=0$. Then as $t \rightarrow 0,\left\{x_{t, n}\right\}$ converges strongly to $p \in \Delta$, which is a unique solution of the VIP (44).

Proof. First let us show that for some $a \in(0,1),\left\{x_{t, n}: t \in\right.$ $(0, a], n \geq 0\}$ is bounded. Indeed, since $\left\{\theta_{t}: t \in(0,1)\right\} \subset$ $[0,1)$ with $\lim _{t \rightarrow 0}\left(\theta_{t} / t\right)=0$, there exists some $a \in(0,1)$ such that $0 \leq \theta_{t} / t<1$ for all $t \in(0, a]$.

Take $p \in \Delta$. Then utilizing Proposition 13, we have

$$
\begin{aligned}
\left\|x_{t, n}-p\right\| & \\
\leq & \left\|f\left(x_{t, n}\right)-p\right\|+(1-t) \\
& \times\left\|\left[G\left(J_{r_{n}} x_{t, n}\right)-\theta_{t} F G\left(J_{r_{n}} x_{t, n}\right)\right]-p\right\| \\
\leq & t \rho\left\|x_{t, n}-p\right\|+t\|f(p)-p\|+(1-t) \\
& \times\left[\left\|\left(I-\theta_{t} F\right) G\left(J_{r_{n}} x_{t, n}\right)-\left(I-\theta_{t} F\right) G\left(J_{r_{n}} p\right)\right\|\right. \\
& \left.+\left\|\left(I-\theta_{t} F\right) G\left(J_{r_{n}} p\right)-p\right\|\right] \\
\leq & t \rho\left\|x_{t, n}-p\right\|+t\|f(p)-p\|+(1-t) \\
& \times\left(1-\theta_{t}\left(1-\sqrt{\frac{1-\delta}{\lambda}}\right)\right)\left\|G\left(J_{r_{n}} x_{t, n}\right)-G\left(J_{r_{n}} p\right)\right\| \\
& +(1-t) \theta_{t}\|F(p)\| \\
\leq & t \rho\left\|x_{t, n}-p\right\|+t\|f(p)-p\|+(1-t) \\
& \times\left(1-\theta_{t}\left(1-\sqrt{\frac{1-\delta}{\lambda}}\right)\right)\left\|J_{r_{n}} x_{t, n}-J_{r_{n}} p\right\| \\
& +\theta_{t}\|F(p)\| \\
\leq & t \rho\left\|x_{t, n}-p\right\|+t\|f(p)-p\|+(1-t) \\
& +\theta_{t}\|F(p)\|, \\
& \times\left(1-\theta_{t}\left(1-\sqrt{\frac{1-\delta}{\lambda}}\right)\right)\left\|x_{t, n}-p\right\|+\theta_{t}\|F(p)\|
\end{aligned}
$$

and, hence, for all $t \in(0, a]$

$$
\begin{aligned}
\left\|x_{t, n}-p\right\| & \leq \frac{1}{1-\rho}\left(\|f(p)-p\|+\frac{\theta_{t}}{t}\|F(p)\|\right) \\
& \leq \frac{1}{1-\rho}(\|f(p)-p\|+\|F(p)\|) .
\end{aligned}
$$

Thus, this implies that $\left\{x_{t, n}: t \in(0, a], n \geq 0\right\}$ is bounded and so are $\left\{f\left(x_{t, n}\right): t \in(0, a], n \geq 0\right\},\left\{J_{r_{n}} x_{t, n}: t \in(0, a], n \geq 0\right\}$, and $\left\{G\left(J_{r_{n}} x_{t, n}\right): t \in(0, a], n \geq 0\right\}$.

Let us show that $\left\|J_{r_{n}} x_{t, n}-G\left(J_{r_{n}} x_{t, n}\right)\right\| \rightarrow 0$ as $t \rightarrow 0$.

Indeed, for simplicity we put $q=\Pi_{C}\left(I-\mu_{2} B_{2}\right) p, \hat{x}_{t, n}=$ $J_{r_{n}} x_{t, n}, u_{t, n}=\Pi_{C}\left(I-\mu_{2} B_{2}\right) \widehat{x}_{t, n}$, and $v_{t, n}=\Pi_{C}\left(I-\mu_{1} B_{1}\right) u_{t, n}$.
Then it is clear that $p=\Pi_{C}\left(I-\mu_{1} B_{1}\right) q$ and $v_{t, n}=G\left(\widehat{x}_{t, n}\right)=$ $G\left(J_{r_{n}} x_{t, n}\right)$. Taking into account $x_{t, n}-p=t\left(f\left(x_{t, n}\right)-p\right)+(1-$ t) $\left[G\left(J_{r_{n}} x_{t, n}\right)-\theta_{t} F G\left(J_{r_{n}} x_{t, n}\right)-p\right]$, we get

$$
\begin{aligned}
\| x_{t, n}- & p \|^{2} \\
= & t\left\langle f\left(x_{t, n}\right)-p, J\left(x_{t, n}-p\right)\right\rangle \\
& +(1-t)\left\langle G\left(J_{r_{n}} x_{t, n}\right)-p, J\left(x_{t, n}-p\right)\right\rangle \\
& -(1-t) \theta_{t}\left\langle F G\left(J_{r_{n}} x_{t, n}\right), J\left(x_{t, n}-p\right)\right\rangle \\
\leq & t\left\langle f\left(x_{t, n}\right)-f(p), J\left(x_{t, n}-p\right)\right\rangle \\
& +t\left\langle f(p)-p, J\left(x_{t, n}-p\right)\right\rangle \\
& +(1-t)\left\|G\left(J_{r_{n}} x_{t, n}\right)-p\right\|\left\|J\left(x_{t, n}-p\right)\right\| \\
& +(1-t) \theta_{t}\left\|F G\left(J_{r_{n}} x_{t, n}\right)\right\|\left\|J\left(x_{t, n}-p\right)\right\| \\
\leq & \rho t\left\|x_{t, n}-p\right\|^{2}+t\|f(p)-p\|\left\|x_{t, n}-p\right\| \\
& +(1-t)\left\|v_{t, n}-p\right\|\left\|x_{t, n}-p\right\| \\
& +\theta_{t}\left\|F v_{t, n}\right\|\left\|x_{t, n}-p\right\| .
\end{aligned}
$$

From Lemma 14 we have

$$
\begin{aligned}
\left\|u_{t, n}-q\right\|^{2} & =\left\|\Pi_{C}\left(\widehat{x}_{t, n}-\mu_{2} B_{2} \widehat{x}_{t, n}\right)-\Pi_{C}\left(p-\mu_{2} B_{2} p\right)\right\|^{2} \\
& \leq\left\|\widehat{x}_{t, n}-p-\mu_{2}\left(B_{2} \widehat{x}_{t, n}-B_{2} p\right)\right\|^{2} \\
& \leq\left\|\widehat{x}_{t, n}-p\right\|^{2}-2 \mu_{2}\left(\alpha_{2}-\kappa^{2} \mu_{2}\right)\left\|B_{2} \widehat{x}_{t, n}-B_{2} p\right\|^{2}, \\
\left\|v_{t, n}-p\right\|^{2} & =\left\|\Pi_{C}\left(u_{t, n}-\mu_{1} B_{1} u_{t, n}\right)-\Pi_{C}\left(q-\mu_{1} B_{1} q\right)\right\|^{2} \\
& \leq\left\|u_{t, n}-q-\mu_{1}\left(B_{1} u_{t, n}-B_{1} q\right)\right\|^{2} \\
& \leq\left\|u_{t, n}-q\right\|^{2}-2 \mu_{1}\left(\alpha_{1}-\kappa^{2} \mu_{1}\right)\left\|B_{1} u_{t, n}-B_{1} q\right\|^{2} .
\end{aligned}
$$

From the last two inequalities, we obtain

$$
\begin{aligned}
\left\|v_{t, n}-p\right\|^{2} \leq & \left\|\widehat{x}_{t, n}-p\right\|^{2}-2 \mu_{2}\left(\alpha_{2}-\kappa^{2} \mu_{2}\right)\left\|B_{2} \widehat{x}_{t, n}-B_{2} p\right\|^{2} \\
& -2 \mu_{1}\left(\alpha_{1}-\kappa^{2} \mu_{1}\right)\left\|B_{1} u_{t, n}-B_{1} q\right\|^{2} \\
\leq & \left\|x_{t, n}-p\right\|^{2}-2 \mu_{2}\left(\alpha_{2}-\kappa^{2} \mu_{2}\right)\left\|B_{2} \widehat{x}_{t, n}-B_{2} p\right\|^{2} \\
& -2 \mu_{1}\left(\alpha_{1}-\kappa^{2} \mu_{1}\right)\left\|B_{1} u_{t, n}-B_{1} q\right\|^{2},
\end{aligned}
$$

which, together with (50), implies that

$$
\begin{aligned}
& \left\|x_{t, n}-p\right\|^{2} \\
& \leq \rho t\left\|x_{t, n}-p\right\|^{2}+t\|f(p)-p\|\left\|x_{t, n}-p\right\| \\
& \quad+(1-t)\left\|v_{t, n}-p\right\|\left\|x_{t, n}-p\right\|+\theta_{t}\left\|F v_{t, n}\right\|\left\|x_{t, n}-p\right\|
\end{aligned}
$$




$$
\begin{aligned}
& \leq \rho t\left\|x_{t, n}-p\right\|^{2}+t\|f(p)-p\|\left\|x_{t, n}-p\right\| \\
& +(1-t) \frac{1}{2}\left(\left\|x_{t, n}-p\right\|^{2}+\left\|v_{t, n}-p\right\|^{2}\right) \\
& +\theta_{t}\left\|F v_{t, n}\right\|\left\|x_{t, n}-p\right\| \\
& \leq \rho t\left\|x_{t, n}-p\right\|^{2}+t\|f(p)-p\|\left\|x_{t, n}-p\right\| \\
& +(1-t) \frac{1}{2}\left\{\left\|x_{t, n}-p\right\|^{2}+\left\|x_{t, n}-p\right\|^{2}\right. \\
& \quad-2 \mu_{2}\left(\alpha_{2}-\kappa^{2} \mu_{2}\right)\left\|B_{2} \widehat{x}_{t, n}-B_{2} p\right\|^{2} \\
& \left.\quad-2 \mu_{1}\left(\alpha_{1}-\kappa^{2} \mu_{1}\right)\left\|B_{1} u_{t, n}-B_{1} q\right\|^{2}\right\} \\
& +\theta_{t}\left\|F v_{t, n}\right\|\left\|x_{t, n}-p\right\| \\
& =(1-t(1-\rho))\left\|x_{t, n}-p\right\|^{2}+t\|f(p)-p\|\left\|x_{t, n}-p\right\| \\
& +(1-t)\left[\mu_{2}\left(\alpha_{2}-\kappa^{2} \mu_{2}\right)\left\|B_{2} \widehat{x}_{t, n}-B_{2} p\right\|^{2}\right. \\
& +\theta_{t}\left\|F v_{t, n}\right\|\left\|x_{t, n}-p\right\| . \\
& +\theta_{t}\left\|F v_{t, n}\right\|\left\|x_{t, n}-p\right\| \\
& +(1-t)\left[\mu_{t, n}-p\left\|^{2}+t\right\| f\left(\alpha_{2}-\kappa^{2} \mu_{2}\right)\left\|B_{2} \hat{x}_{t, n}-B_{2} p\right\|^{2}\right. \\
& \left.\left.+\kappa^{2} \mu_{1}\right)\left\|B_{1} u_{t, n}-B_{1} q\right\|^{2}\right]
\end{aligned}
$$

So it immediately follows that

$$
\begin{aligned}
(1-t) & {\left[\mu_{2}\left(\alpha_{2}-\kappa^{2} \mu_{2}\right)\left\|B_{2} \widehat{x}_{t, n}-B_{2} p\right\|^{2}\right.} \\
& \left.+\mu_{1}\left(\alpha_{1}-\kappa^{2} \mu_{1}\right)\left\|B_{1} u_{t, n}-B_{1} q\right\|^{2}\right] \\
\leq & t\|f(p)-p\|\left\|x_{t, n}-p\right\|+\theta_{t}\left\|F v_{t, n}\right\|\left\|x_{t, n}-p\right\| .
\end{aligned}
$$

Since $0<\mu_{i}<\alpha_{i} / \kappa^{2}$ for $i=1,2$, we have

$$
\begin{aligned}
& \lim _{t \rightarrow 0}\left\|B_{2} \widehat{x}_{t, n}-B_{2} p\right\|=0, \\
& \lim _{t \rightarrow 0}\left\|B_{1} u_{t, n}-B_{1} q\right\|=0 .
\end{aligned}
$$

Utilizing Proposition 3 and Lemma 6, we have

$$
\begin{aligned}
\left\|u_{t, n}-q\right\|^{2}= & \left\|\Pi_{C}\left(\widehat{x}_{t, n}-\mu_{2} B_{2} \widehat{x}_{t, n}\right)-\Pi_{C}\left(p-\mu_{2} B_{2} p\right)\right\|^{2} \\
\leq & \left\langle\widehat{x}_{t, n}-\mu_{2} B_{2} \widehat{x}_{t, n}-\left(p-\mu_{2} B_{2} p\right), J\left(u_{t, n}-q\right)\right\rangle \\
= & \left\langle\widehat{x}_{t, n}-p, J\left(u_{t, n}-q\right)\right\rangle \\
& +\mu_{2}\left\langle B_{2} p-B_{2} \widehat{x}_{t, n}, J\left(u_{t, n}-q\right)\right\rangle
\end{aligned}
$$

$$
\begin{aligned}
\leq & \frac{1}{2}\left[\left\|\hat{x}_{t, n}-p\right\|^{2}+\left\|u_{t, n}-q\right\|^{2}\right. \\
& \left.\quad-g_{1}\left(\left\|\widehat{x}_{t, n}-u_{t, n}-(p-q)\right\|\right)\right] \\
& +\mu_{2}\left\|B_{2} p-B_{2} \widehat{x}_{t, n}\right\|\left\|u_{t, n}-q\right\|,
\end{aligned}
$$

which implies that

$$
\begin{gathered}
\left\|u_{t, n}-q\right\|^{2} \leq \\
\quad\left\|\widehat{x}_{t, n}-p\right\|^{2}-g_{1}\left(\left\|\widehat{x}_{t, n}-u_{t, n}-(p-q)\right\|\right) \\
+2 \mu_{2}\left\|B_{2} p-B_{2} \widehat{x}_{t, n}\right\|\left\|u_{t, n}-q\right\| .
\end{gathered}
$$

In the same way, we derive

$$
\begin{aligned}
\left\|v_{t, n}-p\right\|^{2} & \\
= & \left\|\Pi_{C}\left(u_{t, n}-\mu_{1} B_{1} u_{t, n}\right)-\Pi_{C}\left(q-\mu_{1} B_{1} q\right)\right\|^{2} \\
\leq & \left\langle u_{t, n}-\mu_{1} B_{1} u_{t, n}-\left(q-\mu_{1} B_{1} q\right), J\left(v_{t, n}-p\right)\right\rangle \\
= & \left\langle u_{t, n}-q, J\left(v_{t, n}-p\right)\right\rangle+\mu_{1}\left\langle B_{1} q-B_{1} u_{t, n}, J\left(v_{t, n}-p\right)\right\rangle \\
\leq & \frac{1}{2}\left[\left\|u_{t, n}-q\right\|^{2}+\left\|v_{t, n}-p\right\|^{2}\right. \\
& \left.\quad-g_{2}\left(\left\|u_{t, n}-v_{t, n}+(p-q)\right\|\right)\right] \\
& +\mu_{1}\left\|B_{1} q-B_{1} u_{t, n}\right\|\left\|v_{t, n}-p\right\|,
\end{aligned}
$$

which implies that

$$
\begin{aligned}
\left\|v_{t, n}-p\right\|^{2} \leq & \left\|u_{t, n}-q\right\|^{2}-g_{2}\left(\left\|u_{t, n}-v_{t, n}+(p-q)\right\|\right) \\
& +2 \mu_{1}\left\|B_{1} q-B_{1} u_{t, n}\right\|\left\|v_{t, n}-p\right\| .
\end{aligned}
$$

Substituting (57) for (59), we get

$$
\begin{aligned}
\left\|v_{t, n}-p\right\|^{2} \leq & \left\|\widehat{x}_{t, n}-p\right\|^{2}-g_{1}\left(\left\|\widehat{x}_{t, n}-u_{t, n}-(p-q)\right\|\right) \\
& -g_{2}\left(\left\|u_{t, n}-v_{t, n}+(p-q)\right\|\right) \\
& +2 \mu_{2}\left\|B_{2} p-B_{2} \widehat{x}_{t, n}\right\|\left\|u_{t, n}-q\right\| \\
& +2 \mu_{1}\left\|B_{1} q-B_{1} u_{t, n}\right\|\left\|v_{t, n}-p\right\| \\
\leq & \left\|x_{t, n}-p\right\|^{2}-g_{1}\left(\left\|\widehat{x}_{t, n}-u_{t, n}-(p-q)\right\|\right) \\
& -g_{2}\left(\left\|u_{t, n}-v_{t, n}+(p-q)\right\|\right) \\
& +2 \mu_{2}\left\|B_{2} p-B_{2} \widehat{x}_{t, n}\right\|\left\|u_{t, n}-q\right\| \\
& +2 \mu_{1}\left\|B_{1} q-B_{1} u_{t, n}\right\|\left\|v_{t, n}-p\right\|,
\end{aligned}
$$


which, together with (50), implies that

$$
\begin{aligned}
& \left\|x_{t, n}-p\right\|^{2} \\
& \leq \rho t\left\|x_{t, n}-p\right\|^{2}+t\|f(p)-p\|\left\|x_{t, n}-p\right\| \\
& +(1-t) \frac{1}{2}\left(\left\|x_{t, n}-p\right\|^{2}+\left\|v_{t, n}-p\right\|^{2}\right) \\
& +\theta_{t}\left\|F v_{t, n}\right\|\left\|x_{t, n}-p\right\| \\
& \leq \rho t\left\|x_{t, n}-p\right\|^{2}+t\|f(p)-p\|\left\|x_{t, n}-p\right\| \\
& +(1-t) \frac{1}{2}\left\{\left\|x_{t, n}-p\right\|^{2}+\left\|x_{t, n}-p\right\|^{2}\right. \\
& -g_{1}\left(\left\|\widehat{x}_{t, n}-u_{t, n}-(p-q)\right\|\right) \\
& -g_{2}\left(\left\|u_{t, n}-v_{t, n}+(p-q)\right\|\right) \\
& +2 \mu_{2}\left\|B_{2} p-B_{2} \widehat{x}_{t, n}\right\|\left\|u_{t, n}-q\right\| \\
& \left.+2 \mu_{1}\left\|B_{1} q-B_{1} u_{t, n}\right\|\left\|v_{t, n}-p\right\|\right\} \\
& +\theta_{t}\left\|F v_{t, n}\right\|\left\|x_{t, n}-p\right\| \\
& =(1-t(1-\rho))\left\|x_{t, n}-p\right\|^{2} \\
& +t\|f(p)-p\|\left\|x_{t, n}-p\right\| \\
& -(1-t) \frac{1}{2}\left[g_{1}\left(\left\|\widehat{x}_{t, n}-u_{t, n}-(p-q)\right\|\right)\right. \\
& \left.+g_{2}\left(\left\|u_{t, n}-v_{t, n}+(p-q)\right\|\right)\right] \\
& +(1-t)\left[\mu_{2}\left\|B_{2} p-B_{2} \widehat{x}_{t, n}\right\|\left\|u_{t, n}-q\right\|\right. \\
& \left.+\mu_{1}\left\|B_{1} q-B_{1} u_{t, n}\right\|\left\|v_{t, n}-p\right\|\right] \\
& +\theta_{t}\left\|F v_{t, n}\right\|\left\|x_{t, n}-p\right\| \\
& \leq\left\|x_{t, n}-p\right\|^{2}+t\|f(p)-p\|\left\|x_{t, n}-p\right\| \\
& -(1-t) \frac{1}{2}\left[g_{1}\left(\left\|\widehat{x}_{t, n}-u_{t, n}-(p-q)\right\|\right)\right. \\
& \left.+g_{2}\left(\left\|u_{t, n}-v_{t, n}+(p-q)\right\|\right)\right] \\
& +\mu_{2}\left\|B_{2} p-B_{2} \widehat{x}_{t, n}\right\|\left\|u_{t, n}-q\right\| \\
& +\mu_{1}\left\|B_{1} q-B_{1} u_{t, n}\right\|\left\|v_{t, n}-p\right\| \\
& +\theta_{t}\left\|F v_{t, n}\right\|\left\|x_{t, n}-p\right\| \text {. }
\end{aligned}
$$

So it immediately follows that

$$
\begin{aligned}
(1-t) & \frac{1}{2}\left[g_{1}\left(\left\|\widehat{x}_{t, n}-u_{t, n}-(p-q)\right\|\right)\right. \\
& \left.+g_{2}\left(\left\|u_{t, n}-v_{t, n}+(p-q)\right\|\right)\right] \\
\leq & t\|f(p)-p\|\left\|x_{t, n}-p\right\| \\
& +\mu_{2}\left\|B_{2} p-B_{2} \widehat{x}_{t, n}\right\|\left\|u_{t, n}-q\right\|
\end{aligned}
$$

$$
\begin{aligned}
& +\mu_{1}\left\|B_{1} q-B_{1} u_{t, n}\right\|\left\|v_{t, n}-p\right\| \\
& +\theta_{t}\left\|F v_{t, n}\right\|\left\|x_{t, n}-p\right\| .
\end{aligned}
$$

Hence, from (55) we conclude that

$$
\begin{aligned}
& \lim _{t \rightarrow 0} g_{1}\left(\left\|\widehat{x}_{t, n}-u_{t, n}-(p-q)\right\|\right)=0, \\
& \lim _{t \rightarrow 0} g_{2}\left(\left\|u_{t, n}-v_{t, n}+(p-q)\right\|\right)=0 .
\end{aligned}
$$

Utilizing the properties of $g_{1}$ and $g_{2}$, we get

$$
\begin{aligned}
& \lim _{t \rightarrow 0}\left\|\widehat{x}_{t, n}-u_{t, n}-(p-q)\right\|=0, \\
& \lim _{t \rightarrow 0}\left\|u_{t, n}-v_{t, n}+(p-q)\right\|=0,
\end{aligned}
$$

which leads to

$$
\begin{aligned}
& \left\|\widehat{x}_{t, n}-v_{t, n}\right\| \\
& \leq\left\|\hat{x}_{t, n}-u_{t, n}-(p-q)\right\| \\
& \quad+\left\|u_{t, n}-v_{t, n}+(p-q)\right\| \longrightarrow 0 \quad \text { as } t \longrightarrow 0 .
\end{aligned}
$$

That is,

$$
\lim _{t \rightarrow 0}\left\|J_{r_{n}} x_{t, n}-G\left(J_{r_{n}} x_{t, n}\right)\right\|=\lim _{t \rightarrow 0}\left\|\widehat{x}_{t, n}-v_{t, n}\right\|=0 .
$$

Note that $\operatorname{Fix}\left(J_{r_{n}}\right)=A^{-1}(0)$ for all $n \geq 0$ and that $\left\{x_{t, n}: t \in\right.$ $(0, a], n \geq 0\}$ is bounded and so are $\left\{f\left(x_{t, n}\right): t \in(0, a], n \geq\right.$ $0\},\left\{J_{r_{n}} x_{t, n}: t \in(0, a], n \geq 0\right\}$, and $\left\{G\left(J_{r_{n}} x_{t, n}\right): t \in(0, a], n \geq\right.$ $0\}$. Hence, we have

$$
\begin{aligned}
& \left\|x_{t, n}-G\left(J_{r_{n}} x_{t, n}\right)\right\| \\
& =t\left\|f\left(x_{t, n}\right)-G\left(J_{r_{n}} x_{t, n}\right)-(1-t) \frac{\theta_{t}}{t} F G\left(J_{r_{n}} x_{t, n}\right)\right\| \longrightarrow 0,
\end{aligned}
$$

as $t \rightarrow 0$. Also, observe that

$\left\|x_{t, n}-J_{r_{n}} x_{t, n}\right\| \leq\left\|x_{t, n}-G\left(J_{r_{n}} x_{t, n}\right)\right\|+\left\|G\left(J_{r_{n}} x_{t, n}\right)-J_{r_{n}} x_{t, n}\right\|$.

This, together with (66) and (67), implies that

$$
\lim _{t \rightarrow 0}\left\|x_{t, n}-J_{r_{n}} x_{t, n}\right\|=0 .
$$

Utilizing the nonexpansivity of $G$, we obtain from (67) and (69) that

$$
\begin{aligned}
\left\|x_{t, n}-G x_{t, n}\right\| \leq & \left\|x_{t, n}-G\left(J_{r_{n}} x_{t, n}\right)\right\| \\
& +\left\|G\left(J_{r_{n}} x_{t, n}\right)-G x_{t, n}\right\| \\
\leq & \left\|x_{t, n}-G\left(J_{r_{n}} x_{t, n}\right)\right\| \\
& +\left\|J_{r_{n}} x_{t, n}-x_{t, n}\right\| \longrightarrow 0, \text { as } t \longrightarrow 0 .
\end{aligned}
$$


Since $r_{n} \geq \varepsilon$ for all $n$, utilizing Lemma 9 we have

$$
\left\|x_{t, n}-J_{\varepsilon} x_{t, n}\right\| \leq 2\left\|x_{t, n}-J_{r_{n}} x_{t, n}\right\| \longrightarrow 0, \quad \text { as } t \longrightarrow 0
$$

For any integer $n \geq 0$, for simplicity put $w_{t}=x_{t, n}$ for all $t \in(0, a]$. Now let $\left\{t_{k}\right\}$ be a sequence in $(0, a]$ that converges to 0 as $k \rightarrow \infty$ and define a function $g$ on $C$ by

$$
g(w)=\mu_{k} \frac{1}{2}\left\|w_{t_{k}}-w\right\|^{2}, \quad \forall w \in C
$$

where $\mu$ is a Banach limit. Define the set

$$
K:=\{w \in C: g(w)=\min \{g(y): y \in C\}\}
$$

and the mapping

$$
W x=(1-\theta) J_{\varepsilon} x+\theta G x, \quad \forall x \in C,
$$

where $\theta$ is a constant in $(0,1)$. Then by Lemma 10 , we know that $\operatorname{Fix}(W)=\operatorname{Fix}\left(J_{\varepsilon}\right) \cap \operatorname{Fix}(G)=\Delta$. We observe that

$$
\begin{aligned}
\left\|w_{t}-W w_{t}\right\| & =\left\|(1-\theta)\left(w_{t}-J_{\varepsilon} w_{t}\right)+\theta\left(w_{t}-G w_{t}\right)\right\| \\
& \leq(1-\theta)\left\|w_{t}-J_{\varepsilon} w_{t}\right\|+\theta\left\|w_{t}-G w_{t}\right\| .
\end{aligned}
$$

So from (70) and (71) we obtain

$$
\lim _{n \rightarrow \infty}\left\|w_{t}-W w_{t}\right\|=0
$$

Since $X$ is a uniformly smooth Banach space, $K$ is a nonempty bounded closed convex subset of $C$; for more details, see [43]. We claim that $K$ is also invariant under the nonexpansive mapping $W$. Indeed, noticing (76), we have for $w \in K$

$$
\begin{aligned}
g(W w) & =\mu_{k} \frac{1}{2}\left\|w_{t_{k}}-W w\right\|^{2}=\mu_{k} \frac{1}{2}\left\|W w_{t_{k}}-W w\right\|^{2} \\
& \leq \mu_{k} \frac{1}{2}\left\|w_{t_{k}}-w\right\|^{2}=g(w) .
\end{aligned}
$$

Since every nonempty closed bounded convex subset of uniformly smooth Banach space $X$ has the fixed point property for nonexpansive mappings and $W$ is a nonexpansive mapping of $K, W$ has a fixed point in $K$, say $p$. Utilizing Lemma 11, we get

$$
\mu_{k}\left\langle x-p, J\left(w_{t_{k}}-p\right)\right\rangle \leq 0, \quad \forall x \in C
$$

Putting $x=f(p)$ we have

$$
\mu_{k}\left\langle f(p)-p, J\left(w_{t_{k}}-p\right)\right\rangle \leq 0, \quad \forall x \in C
$$

Since $w_{t_{k}}-p=t_{k}\left(f\left(w_{t_{k}}\right)-p\right)+\left(1-t_{k}\right)\left[G\left(J_{r_{n}} w_{t_{k}}\right)-\right.$ $\left.\theta_{t_{k}} F G\left(J_{r_{n}} w_{t_{k}}\right)-p\right]$, we get

$$
\begin{aligned}
\left\|w_{t_{k}}-p\right\|^{2} & \\
= & t_{k}\left\langle f\left(w_{t_{k}}\right)-p, J\left(w_{t_{k}}-p\right)\right\rangle \\
& +\left(1-t_{k}\right)\left\langle G\left(J_{r_{n}} w_{t_{k}}\right)-p, J\left(w_{t_{k}}-p\right)\right\rangle \\
& -\left(1-t_{k}\right) \theta_{t_{k}}\left\langle F G\left(J_{r_{n}} w_{t_{k}}\right), J\left(w_{t_{k}}-p\right)\right\rangle \\
\leq & t_{k}\left\langle f\left(w_{t_{k}}\right)-f(p), J\left(w_{t_{k}}-p\right)\right\rangle \\
& +t_{k}\left\langle f(p)-p, J\left(w_{t_{k}}-p\right)\right\rangle \\
& +\left(1-t_{k}\right)\left\|G\left(J_{r_{n}} w_{t_{k}}\right)-p\right\|\left\|J\left(w_{t_{k}}-p\right)\right\| \\
& +\left(1-t_{k}\right) \theta_{t_{k}}\left\|F G\left(J_{r_{n}} w_{t_{k}}\right)\right\|\left\|J\left(w_{t_{k}}-p\right)\right\| \\
\leq & \rho t_{k}\left\|w_{t_{k}}-p\right\|^{2}+t_{k}\left\langle f(p)-p, J\left(w_{t_{k}}-p\right)\right\rangle \\
& +\left(1-t_{k}\right)\left\|w_{t_{k}}-p\right\|^{2} \\
& +\theta_{t_{k}}\left\|F G\left(J_{r_{n}} w_{t_{k}}\right)\right\|\left\|w_{t_{k}}-p\right\| .
\end{aligned}
$$

It follows that

$$
\begin{aligned}
\left\|w_{t_{k}}-p\right\|^{2} \leq \frac{1}{1-\rho}[ & \left\langle f(p)-p, J\left(w_{t_{k}}-p\right)\right\rangle \\
& \left.+\frac{\theta_{t_{k}}}{t_{k}}\left\|F G\left(J_{r_{n}} w_{t_{k}}\right)\right\|\left\|w_{t_{k}}-p\right\|\right] .
\end{aligned}
$$

Since $\lim _{k \rightarrow \infty}\left(\theta_{t_{k}} / t_{k}\right)=0$, from (79) and the boundedness of sequences $\left\{F G\left(J_{r_{n}} w_{t_{k}}\right)\right\},\left\{w_{t_{k}}\right\}$, it follows that

$$
\begin{gathered}
\mu_{k}\left\|w_{t_{k}}-p\right\|^{2} \\
\leq \frac{1}{1-\rho} \mu_{k}\left[\left\langle f(p)-p, J\left(w_{t_{k}}-p\right)\right\rangle\right. \\
\left.\quad+\frac{\theta_{t_{k}}}{t_{k}}\left\|F G\left(J_{r_{n}} w_{t_{k}}\right)\right\|\left\|w_{t_{k}}-p\right\|\right] \\
=\frac{1}{1-\rho}\left[\begin{array}{c}
\mu_{k}\left\langle f(p)-p, J\left(w_{t_{k}}-p\right)\right\rangle \\
\left.+\mu_{k}\left(\frac{\theta_{t_{k}}}{t_{k}}\left\|F G\left(J_{r_{n}} w_{t_{k}}\right)\right\|\left\|w_{t_{k}}-p\right\|\right)\right] \leq 0 .
\end{array}\right.
\end{gathered}
$$

Therefore, it is known that for any sequence $\left\{w_{t_{k}}\right\}$ in $\left\{w_{t}\right.$ : $t \in(0, a]\}$ there exists a subsequence which is still denoted by $\left\{w_{t_{k}}\right\}$ that converges strongly to some fixed point $p$ of $W$. To prove that the net $\left\{w_{t}: t \in(0, a]\right\}$ converges strongly to $p$ as $t \rightarrow 0$, suppose that there exists another subsequence $\left\{w_{s_{k}}\right\} \subset\left\{w_{t}\right\}$ such that $w_{s_{k}} \rightarrow q$ as $s_{k} \rightarrow 0$, and then we also have $q \in \operatorname{Fix}(W)=A^{-1}(0) \cap \Omega=: \Delta$ due to (76). 
Since the sets $\left\{w_{t}-u: t \in(0, a]\right\}$ and $\left\{w_{t}-f\left(w_{t}\right):\right.$ $t \in(0, a]\}$ are bounded and the duality map $J$ is single-valued and norm-to-norm uniformly continuous on bounded sets of uniformly smooth Banach space $X$, for any $u \in \Delta=A^{-1}(0) \cap$ $\Omega$, from $w_{s_{k}} \rightarrow q\left(s_{k} \rightarrow 0\right)$ we obtain

$$
\begin{gathered}
\left\|(I-f) w_{s_{k}}-(I-f) q\right\| \longrightarrow 0 \quad\left(s_{k} \rightarrow 0\right), \\
\left|\left\langle w_{s_{k}}-f\left(w_{s_{k}}\right), J\left(w_{s_{k}}-u\right)\right\rangle-\langle(I-f) q, J(q-u)\rangle\right| \\
=\mid\left\langle(I-f) w_{s_{k}}-(I-f) q, J\left(w_{s_{k}}-u\right)\right\rangle \\
\quad+\left\langle(I-f) q, J\left(w_{s_{k}}-u\right)-J(q-u)\right\rangle \mid \\
\leq\left\|(I-f) w_{s_{k}}-(I-f) q\right\|\left\|w_{s_{k}}-u\right\| \\
\quad+\left|\left\langle(I-f) q, J\left(w_{s_{k}}-u\right)-J(q-u)\right\rangle\right| \longrightarrow 0,
\end{gathered}
$$

as $s_{k} \rightarrow 0$. Therefore,

$$
\langle(I-f) q, J(q-u)\rangle=\lim _{s_{k} \rightarrow 0}\left\langle w_{s_{k}}-f\left(w_{s_{k}}\right), J\left(w_{s_{k}}-u\right)\right\rangle .
$$

Since $u \in \Delta=A^{-1}(0) \cap \Omega$,

$$
\begin{aligned}
\| x_{t, n}- & {\left[(1-t) u+t f\left(x_{t, n}\right)\right] \|^{2} } \\
= & \|(1-t) G\left(J_{r_{n}} x_{t, n}\right)+t f\left(x_{t, n}\right)-(1-t) u-t f\left(x_{t, n}\right) \\
& -(1-t) \theta_{t} F G\left(J_{r_{n}} x_{t, n}\right) \|^{2} \\
\leq & {\left[(1-t)\left\|G\left(J_{r_{n}} x_{t, n}\right)-u\right\|+(1-t) \theta_{t}\left\|F G\left(J_{r_{n}} x_{t, n}\right)\right\|\right]^{2} } \\
\leq & {\left[(1-t)\left\|x_{t, n}-u\right\|+(1-t) \theta_{t}\left\|F G\left(J_{r_{n}} x_{t, n}\right)\right\|\right]^{2} } \\
= & (1-t)^{2}\left\|x_{t, n}-u\right\|^{2}+2(1-t)^{2} \theta_{t}\left\|x_{t, n}-u\right\|\left\|F G\left(J_{r_{n}} x_{t, n}\right)\right\| \\
& +(1-t)^{2} \theta_{t}^{2}\left\|F G\left(J_{r_{n}} x_{t, n}\right)\right\|^{2} .
\end{aligned}
$$

Utilizing Lemma 5, we have

$$
\begin{aligned}
\| x_{t, n}- & {\left[(1-t) u+t f\left(x_{t, n}\right)\right] \|^{2} } \\
= & \left\|(1-t)\left(x_{t, n}-u\right)+t\left(x_{t, n}-f\left(x_{t, n}\right)\right)\right\|^{2} \\
\geq & (1-t)^{2}\left\|x_{t, n}-u\right\|^{2} \\
& +2 t(1-t)\left\langle x_{t, n}-f\left(x_{t, n}\right), J\left(x_{t, n}-u\right)\right\rangle .
\end{aligned}
$$

Consequently, from the last two inequalities we deduce that

$$
\begin{aligned}
2 t(1-t) & \left\langle x_{t, n}-f\left(x_{t, n}\right), J\left(x_{t, n}-u\right)\right\rangle \\
\leq & 2(1-t)^{2} \theta_{t}\left\|x_{t, n}-u\right\|\left\|F G\left(J_{r_{n}} x_{t, n}\right)\right\| \\
& +(1-t)^{2} \theta_{t}^{2}\left\|F G\left(J_{r_{n}} x_{t, n}\right)\right\|^{2},
\end{aligned}
$$

and hence

$$
\begin{aligned}
\left\langle x_{t, n}-\right. & \left.f\left(x_{t, n}\right), J\left(x_{t, n}-u\right)\right\rangle \\
\leq & (1-t) \frac{\theta_{t}}{t}\left\|x_{t, n}-u\right\|\left\|F G\left(J_{r_{n}} x_{t, n}\right)\right\| \\
& +(1-t) \frac{\theta_{t}^{2}}{2 t}\left\|F G\left(J_{r_{n}} x_{t, n}\right)\right\|^{2} .
\end{aligned}
$$

Noticing (84), from $\lim _{t \rightarrow 0}\left(\theta_{t} / t\right)=0$ and the boundedness of sequences $\left\{F G\left(J_{r_{n}} x_{t, n}\right)\right\},\left\{x_{t, n}\right\}$, we conclude that

$$
\langle(I-f) q, J(q-u)\rangle \leq 0 .
$$

Interchanging $p$ and $u$ leads to

$$
\langle(I-f) q, J(q-p)\rangle \leq 0 .
$$

Interchanging $q$ and $p$ leads to

$$
\langle(I-f) p, J(p-q)\rangle \leq 0 .
$$

This implies that

$$
\langle(q-p)-(f(q)-f(p)), J(p-q)\rangle \leq 0,
$$

and hence

$$
\|q-p\|^{2} \leq \rho\|q-p\|^{2}
$$

Taking into account $\rho \in(0,1)$, we obtain $p=q$. Furthermore, by the careful analysis of the above proof, we can readily see that $p$ is also a unique solution of the VIP:

$$
\langle(I-f) p, J(p-u)\rangle \leq 0, \quad \forall u \in \Delta .
$$

This completes the proof.

Remark 17. In the assertion of Theorem 16, "as $t \rightarrow 0,\left\{x_{t, n}\right\}$ converges strongly to $p \in \Delta ;$; this $p$ does not depend on $n$. Indeed, it is known that there holds the condition that $\left\{r_{n}\right\} \subset$ $[\varepsilon, \infty)$ for some $\varepsilon>0$. Moreover, in the proof of Theorem 16 it can be readily seen that $p$ is first found out as a fixed point of the nonexpansive self-mapping $W$ of $K$. This shows that $p$ depends on neither $n$ nor $t$.

Theorem 18. Let $X$ be a uniformly convex and 2-uniformly smooth Banach space and let $A$ be an $m$-accretive operator in $X$ such that $C=\overline{D(A)}$ is convex. Let $\Pi_{C}$ be a sunny nonexpansive retraction from $X$ onto $C$. Let the mapping $B_{i}: C \rightarrow X$ be $\alpha_{i}$-inverse strongly accretive for $i=1,2, f: X \rightarrow C$ be a contractive map with coefficient $\rho \in(0,1)$, and let $F: X \rightarrow$ $X$ be $\delta$-strongly accretive and $\lambda$-strictly pseudocontractive with $\delta+\lambda>1$. Assume that $\Delta=A^{-1}(0) \cap \Omega \neq \emptyset$, where $\Omega$ is the fixed point set of the mapping $G=\Pi_{C}\left(I-\mu_{1} B_{1}\right) \Pi_{C}\left(I-\mu_{2} B_{2}\right)$ with $0<\mu_{i}<\alpha_{i} / \kappa^{2}$ for $i=1,2$. Given sequences $\left\{\lambda_{n}\right\}_{n=0}^{\infty},\left\{\mu_{n}\right\}_{n=0}^{\infty}$ in $[0,1],\left\{\alpha_{n}\right\}_{n=0}^{\infty},\left\{\beta_{n}\right\}_{n=0}^{\infty}$ in $(0,1]$, and $\left\{r_{n}\right\}_{n=0}^{\infty}$ in $[\varepsilon, \infty)$ for some $\varepsilon>0$, suppose that there hold the following conditions:

(i) $\lim _{n \rightarrow \infty} \beta_{n}=0$ and $\sum_{n=0}^{\infty} \beta_{n}=\infty$,

(ii) $\lim _{n \rightarrow \infty}\left(\lambda_{n} \mu_{n}\right) / \beta_{n}=0$, 
(iii) $\left\{\alpha_{n}\right\} \subset[a, b]$ for some $a, b \in(0,1)$,

(iv) $\sum_{n=0}^{\infty}\left|\alpha_{n+1}-\alpha_{n}\right|<\infty, \sum_{n=0}^{\infty}\left|\beta_{n+1}-\beta_{n}\right|<\infty$, $\sum_{n=0}^{\infty}\left|\lambda_{n+1}-\lambda_{n}\right|<\infty, \sum_{n=0}^{\infty}\left|\mu_{n+1}-\mu_{n}\right|<\infty$ and $\sum_{n=0}^{\infty}\left|r_{n+1}-r_{n}\right|<\infty$.

Then for any given point $x_{0} \in X$, the sequence $\left\{x_{n}\right\}$ generated by

$$
\begin{aligned}
& y_{n}=\alpha_{n} x_{n}+\left(1-\alpha_{n}\right) G\left(J_{r_{n}} x_{n}\right), \\
& x_{n+1}=\beta_{n} f\left(x_{n}\right)+\left(1-\beta_{n}\right)\left[G\left(J_{r_{n}} y_{n}\right)-\lambda_{n} \mu_{n} F G\left(J_{r_{n}} y_{n}\right)\right], \\
& \forall n \geq 0,
\end{aligned}
$$

converges strongly to $p \in \Delta$, which is a unique solution of the VIP (44).

Proof. First, let us show that $\left\{x_{n}\right\}$ is bounded. Indeed, taking a fixed $u \in \Delta$ arbitrarily, we have

$$
\begin{aligned}
\left\|y_{n}-u\right\| & =\left\|\alpha_{n} x_{n}+\left(1-\alpha_{n}\right) G\left(J_{r_{n}} x_{n}\right)-u\right\| \\
& \leq \alpha_{n}\left\|x_{n}-u\right\|+\left(1-\alpha_{n}\right)\left\|G\left(J_{r_{n}} x_{n}\right)-u\right\| \\
& \leq \alpha_{n}\left\|x_{n}-u\right\|+\left(1-\alpha_{n}\right)\left\|J_{r_{n}} x_{n}-u\right\| \\
& \leq \alpha_{n}\left\|x_{n}-u\right\|+\left(1-\alpha_{n}\right)\left\|x_{n}-u\right\| \\
& =\left\|x_{n}-u\right\| .
\end{aligned}
$$

So $\left\|y_{n}-u\right\| \leq\left\|x_{n}-u\right\|$ for all $n \geq 0$. Thus, by Proposition 13 (iii), we have

$$
\begin{aligned}
& \left\|x_{n+1}-u\right\| \\
& =\left\|\beta_{n} f\left(x_{n}\right)+\left(1-\beta_{n}\right)\left[G\left(J_{r_{n}} y_{n}\right)-\lambda_{n} \mu_{n} F G\left(J_{r_{n}} y_{n}\right)\right]-u\right\| \\
& \leq \beta_{n}\left\|f\left(x_{n}\right)-u\right\|+\left(1-\beta_{n}\right) \\
& \quad \times\left\|\lambda_{n}\left(I-\mu_{n} F\right) G\left(J_{r_{n}} y_{n}\right)+\left(1-\lambda_{n}\right) G\left(J_{r_{n}} y_{n}\right)-u\right\| \\
& \leq \beta_{n} \rho\left\|x_{n}-u\right\|+\beta_{n}\|f(u)-u\| \\
& \quad+\left(1-\beta_{n}\right)\left[\lambda_{n}\left\|\left(I-\mu_{n} F\right) G\left(J_{r_{n}} y_{n}\right)-u\right\|\right. \\
& \left.\quad+\left(1-\lambda_{n}\right)\left\|G\left(J_{r_{n}} y_{n}\right)-u\right\|\right] \\
& \quad \beta_{n} \rho\left\|x_{n}-u\right\|+\beta_{n}\|f(u)-u\| \\
& +\left(1-\beta_{n}\right)\left[\lambda_{n}\left\|\left(I-\mu_{n} F\right) G\left(J_{r_{n}} y_{n}\right)-\left(I-\mu_{n} F\right) u\right\|\right. \\
& \quad+\lambda_{n}\left\|\left(I-\mu_{n} F\right) u-u\right\| \\
& \left.\quad+\left(1-\lambda_{n}\right)\left\|G\left(J_{r_{n}} y_{n}\right)-u\right\|\right]
\end{aligned}
$$

$$
\begin{aligned}
& +\left(1-\beta_{n}\right)\left[\lambda_{n}\left(1-\mu_{n}\left(1-\sqrt{\frac{1-\delta}{\lambda}}\right)\right)\left\|G\left(J_{r_{n}} y_{n}\right)-u\right\|\right. \\
& \left.+\lambda_{n} \mu_{n}\|F u\|+\left(1-\lambda_{n}\right)\left\|G\left(J_{r_{n}} y_{n}\right)-u\right\|\right] \\
& =\beta_{n} \rho\left\|x_{n}-u\right\|+\beta_{n}\|f(u)-u\| \\
& +\left(1-\beta_{n}\right)\left[\left(1-\lambda_{n} \mu_{n}\left(1-\sqrt{\frac{1-\delta}{\lambda}}\right)\right)\left\|G\left(J_{r_{n}} y_{n}\right)-u\right\|\right. \\
& \left.+\lambda_{n} \mu_{n}\|F u\|\right] \\
& =\beta_{n} \rho\left\|x_{n}-u\right\|+\beta_{n}\|f(u)-u\| \\
& +\left(1-\beta_{n}\right)\left[\left(1-\lambda_{n} \mu_{n}\left(1-\sqrt{\frac{1-\delta}{\lambda}}\right)\right)\left\|G\left(J_{r_{n}} y_{n}\right)-u\right\|\right. \\
& \left.+\lambda_{n} \mu_{n}\left(1-\sqrt{\frac{1-\delta}{\lambda}}\right)\left(1-\sqrt{\frac{1-\delta}{\lambda}}\right)^{-1}\|F u\|\right] \\
& \leq \beta_{n} \rho\left\|x_{n}-u\right\|+\beta_{n}\|f(u)-u\| \\
& +\left(1-\beta_{n}\right) \max \left\{\left\|G\left(J_{r_{n}} y_{n}\right)-u\right\|,\left(1-\sqrt{\frac{1-\delta}{\lambda}}\right)^{-1}\|F u\|\right\} \\
& \leq \beta_{n} \rho\left\|x_{n}-u\right\|+\beta_{n}\|f(u)-u\| \\
& +\left(1-\beta_{n}\right) \max \left\{\left\|y_{n}-u\right\|,\left(1-\sqrt{\frac{1-\delta}{\lambda}}\right)^{-1}\|F u\|\right\} \\
& \leq \beta_{n} \rho\left\|x_{n}-u\right\|+\beta_{n}\|f(u)-u\| \\
& +\left(1-\beta_{n}\right) \max \left\{\left\|x_{n}-u\right\|,\left(1-\sqrt{\frac{1-\delta}{\lambda}}\right)^{-1}\|F u\|\right\} \\
& \leq \beta_{n}\|f(u)-u\|+\left(1-(1-\rho) \beta_{n}\right) \\
& \times \max \left\{\left\|x_{n}-u\right\|,\left(1-\sqrt{\frac{1-\delta}{\lambda}}\right)^{-1}\|F u\|\right\} \\
& =(1-\rho) \beta_{n} \frac{\|f(u)-u\|}{1-\rho}+\left(1-(1-\rho) \beta_{n}\right) \\
& \times \max \left\{\left\|x_{n}-u\right\|,\left(1-\sqrt{\frac{1-\delta}{\lambda}}\right)^{-1}\|F u\|\right\} \\
& \leq \max \left\{\left\|x_{n}-u\right\|, \frac{\|f(u)-u\|}{1-\rho},\left(1-\sqrt{\frac{1-\delta}{\lambda}}\right)^{-1}\|F u\|\right\} .
\end{aligned}
$$


By induction,

$$
\begin{aligned}
& \left\|x_{n}-u\right\| \\
& \leq \max \left\{\left\|x_{0}-u\right\|, \frac{\|f(u)-u\|}{1-\rho},\left(1-\sqrt{\frac{1-\delta}{\lambda}}\right)^{-1}\|F u\|\right\},
\end{aligned}
$$$$
\forall n \geq 0 \text {. }
$$

Thus, $\left\{x_{n}\right\}$ is bounded and so is $\left\{y_{n}\right\}$. Because $G$ and $J_{r_{n}}$ are nonexpansive for all $n \geq 0, f$ is contractive, and $F$ is Lipschitzian, $\left\{J_{r_{n}} x_{n}\right\},\left\{J_{r_{n}} y_{n}\right\},\left\{G\left(J_{r_{n}} x_{n}\right)\right\},\left\{G\left(J_{r_{n}} y_{n}\right)\right\},\left\{f\left(x_{n}\right)\right\}$, and $\left\{F G\left(J_{r_{n}} y_{n}\right)\right\}^{n}$ are bounded. From conditions (i), (ii) we have

$$
\begin{aligned}
\left\|x_{n+1}-G\left(J_{r_{n}} y_{n}\right)\right\| \\
\leq \beta_{n}\left\|f\left(x_{n}\right)-G\left(J_{r_{n}} y_{n}\right)\right\| \\
\quad+\left(1-\beta_{n}\right)\left\|G\left(J_{r_{n}} y_{n}\right)-\lambda_{n} \mu_{n} F G\left(J_{r_{n}} y_{n}\right)-G\left(J_{r_{n}} y_{n}\right)\right\| \\
=\beta_{n}\left\|f\left(x_{n}\right)-G\left(J_{r_{n}} y_{n}\right)\right\| \\
\quad+\left(1-\beta_{n}\right) \lambda_{n} \mu_{n}\left\|F G\left(J_{r_{n}} y_{n}\right)\right\| \longrightarrow 0 \text { as } n \longrightarrow \infty .
\end{aligned}
$$

Now, we claim that

$$
\left\|x_{n+1}-x_{n}\right\| \longrightarrow 0 \text { as } n \longrightarrow \infty \text {. }
$$

In order to prove (100), we estimate $\left\|x_{n+1}-x_{n}\right\|$ first. From (95) we have

$$
\begin{gathered}
y_{n}=\alpha_{n} x_{n}+\left(1-\alpha_{n}\right) G\left(J_{r_{n}} x_{n}\right), \\
y_{n-1}=\alpha_{n-1} x_{n-1}+\left(1-\alpha_{n-1}\right) G\left(J_{r_{n-1}} x_{n-1}\right) .
\end{gathered}
$$

Simple calculations show that

$$
\begin{aligned}
y_{n}-y_{n-1}= & \left(1-\alpha_{n}\right)\left(G\left(J_{r_{n}} x_{n}\right)-G\left(J_{r_{n-1}} x_{n-1}\right)\right) \\
& +\alpha_{n}\left(x_{n}-x_{n-1}\right) \\
& +\left(x_{n-1}-G\left(J_{r_{n-1}} x_{n-1}\right)\right)\left(\alpha_{n}-\alpha_{n-1}\right) .
\end{aligned}
$$

It follows that

$$
\begin{aligned}
\left\|y_{n}-y_{n-1}\right\| \leq & \left(1-\alpha_{n}\right)\left\|G\left(J_{r_{n}} x_{n}\right)-G\left(J_{r_{n-1}} x_{n-1}\right)\right\| \\
& +\alpha_{n}\left\|x_{n}-x_{n-1}\right\| \\
& +\left\|x_{n-1}-G\left(J_{r_{n-1}} x_{n-1}\right)\right\|\left|\alpha_{n}-\alpha_{n-1}\right| \\
\leq & \left(1-\alpha_{n}\right)\left\|J_{r_{n}} x_{n}-J_{r_{n-1}} x_{n-1}\right\| \\
& +\alpha_{n}\left\|x_{n}-x_{n-1}\right\| \\
& +\left\|x_{n-1}-G\left(J_{r_{n-1}} x_{n-1}\right)\right\|\left|\alpha_{n}-\alpha_{n-1}\right| .
\end{aligned}
$$

On the other hand, if $r_{n-1} \leq r_{n}$, using the resolvent identity in Lemma 8

$$
J_{r_{n}} x_{n}=J_{r_{n-1}}\left(\frac{r_{n-1}}{r_{n}} x_{n}+\left(1-\frac{r_{n-1}}{r_{n}}\right) J_{r_{n}} x_{n}\right),
$$

we get

$$
\begin{aligned}
\left\|J_{r_{n}} x_{n}-J_{r_{n-1}} G_{n-1}\right\| \\
\quad=\left\|J_{r_{n-1}}\left(\frac{r_{n-1}}{r_{n}} x_{n}+\left(1-\frac{r_{n-1}}{r_{n}}\right) J_{r_{n}} x_{n}\right)-J_{r_{n-1}} x_{n-1}\right\| \\
\quad \leq \frac{r_{n-1}}{r_{n}}\left\|x_{n}-x_{n-1}\right\|+\left(1-\frac{r_{n-1}}{r_{n}}\right)\left\|J_{r_{n}} x_{n}-x_{n-1}\right\| \\
\leq\left\|x_{n}-x_{n-1}\right\|+\frac{r_{n}-r_{n-1}}{r_{n}}\left\|J_{r_{n}} x_{n}-x_{n-1}\right\| \\
\leq\left\|x_{n}-x_{n-1}\right\|+\frac{1}{\varepsilon}\left|r_{n}-r_{n-1}\right|\left\|J_{r_{n}} x_{n}-x_{n-1}\right\| .
\end{aligned}
$$

If $r_{n} \leq r_{n-1}$, it is easy to see that

$$
\begin{aligned}
& \left\|J_{r_{n}} x_{n}-J_{r_{n-1}} x_{n-1}\right\| \\
& \quad \leq\left\|x_{n-1}-x_{n}\right\|+\frac{1}{\varepsilon}\left|r_{n-1}-r_{n}\right|\left\|J_{r_{n-1}} x_{n-1}-x_{n}\right\| .
\end{aligned}
$$

So combining the above cases we obtain

$$
\begin{aligned}
\| J_{r_{n}} x_{n} & -J_{r_{n-1}} x_{n-1} \| \\
\leq & \left\|x_{n-1}-x_{n}\right\|+\frac{\left|r_{n-1}-r_{n}\right|}{\varepsilon} \\
& \quad \sup _{n \geq 1}\left\{\left\|J_{r_{n}} x_{n}-x_{n-1}\right\|+\left\|J_{r_{n-1}} x_{n-1}-x_{n}\right\|\right\},
\end{aligned}
$$

$\forall n \geq 1$

In the similar way we can derive

$$
\begin{aligned}
\| J_{r_{n}} y_{n} & -J_{r_{n-1}} y_{n-1} \| \\
\leq & \left\|y_{n-1}-y_{n}\right\|+\frac{\left|r_{n-1}-r_{n}\right|}{\varepsilon} \\
& \quad \sup _{n \geq 1}\left\{\left\|J_{r_{n}} y_{n}-y_{n-1}\right\|+\left\|J_{r_{n-1}} y_{n-1}-y_{n}\right\|\right\},
\end{aligned}
$$

$\forall n \geq 1$

Therefore, we have

$$
\begin{aligned}
& \left\|J_{r_{n}} x_{\mathrm{n}}-J_{r_{n-1}} x_{n-1}\right\| \leq\left\|x_{n-1}-x_{n}\right\|+\left|r_{n-1}-r_{n}\right| M_{0}, \\
& \left\|J_{r_{n}} y_{n}-J_{r_{n-1}} y_{n-1}\right\| \leq\left\|y_{n-1}-y_{n}\right\|+\left|r_{n-1}-r_{n}\right| M_{0},
\end{aligned}
$$

for all $n \geq 1$, where $\sup _{n \geq 1}\left\{(1 / \varepsilon)\left(\left\|J_{r_{n}} x_{n}-x_{n-1}\right\|+\| J_{r_{n-1}} x_{n-1}-\right.\right.$ $\left.\left.x_{n} \|\right)\right\} \leq M_{0}$ and $\sup _{n \geq 1}\left\{(1 / \varepsilon)\left(\left\|J_{r_{n}} y_{n}-y_{n-1}\right\|+\| J_{r_{n-1}}^{n-1} y_{n-1}-\right.\right.$ $\left.\left.y_{n} \|\right)\right\} \leq \mathrm{M}_{0}$ for some $M_{0}>0$. 
Substituting (109) for (103), we obtain

$$
\begin{aligned}
\left\|y_{n}-y_{n-1}\right\| \leq & \left(1-\alpha_{n}\right)\left\|J_{r_{n}} x_{n}-J_{r_{n-1}} x_{n-1}\right\| \\
& +\alpha_{n}\left\|x_{n}-x_{n-1}\right\| \\
& +\left\|x_{n-1}-G\left(J_{r_{n-1}} x_{n-1}\right)\right\|\left|\alpha_{n}-\alpha_{n-1}\right| \\
\leq & \left(1-\alpha_{n}\right)\left(\left\|x_{n}-x_{n-1}\right\|+\left|r_{n}-r_{n-1}\right| M_{0}\right) \\
& +\alpha_{n}\left\|x_{n}-x_{n-1}\right\| \\
& +\left\|x_{n-1}-G\left(J_{r_{n-1}} x_{n-1}\right)\right\|\left|\alpha_{n}-\alpha_{n-1}\right| \\
\leq & \left\|x_{n}-x_{n-1}\right\| \\
& +M_{1}\left(\left|r_{n}-r_{n-1}\right|+\left|\alpha_{n}-\alpha_{n-1}\right|\right)
\end{aligned}
$$

where $\sup _{n \geq 0}\left\{M_{0},\left\|x_{n}-G\left(J_{r_{n}} x_{n}\right)\right\|\right\} \leq M_{1}$ for some constant $M_{1}>0$.

In the meantime, it follows from (95) that

$$
\begin{array}{rl}
x_{n+1}=\beta_{n} & f\left(x_{n}\right)+\left(1-\beta_{n}\right)\left[G\left(J_{r_{n}} y_{n}\right)-\lambda_{n} \mu_{n} F G\left(J_{r_{n}} y_{n}\right)\right], \\
x_{n}= & \beta_{n-1} f\left(x_{n-1}\right)+\left(1-\beta_{n-1}\right) \\
& \times\left[G\left(J_{r_{n-1}} y_{n-1}\right)-\lambda_{n-1} \mu_{n-1} F G\left(J_{r_{n-1}} y_{n-1}\right)\right] .
\end{array}
$$

Simple calculations show that

$$
\begin{aligned}
x_{n+1} & -x_{n} \\
= & \left(\beta_{n}-\beta_{n-1}\right) f\left(x_{n-1}\right)+\beta_{n}\left(f\left(x_{n}\right)-f\left(x_{n-1}\right)\right) \\
& +\left(\beta_{n-1}-\beta_{n}\right)\left(I-\lambda_{n-1} \mu_{n-1} F\right) G\left(J_{r_{n-1}} y_{n-1}\right) \\
& +\left(1-\beta_{n}\right)\left[\left(I-\lambda_{n} \mu_{n} F\right) G\left(J_{r_{n}} y_{n}\right)\right. \\
& \left.-\left(I-\lambda_{n-1} \mu_{n-1} F\right) G\left(J_{r_{n-1}} y_{n-1}\right)\right] \\
= & \left(\beta_{n}-\beta_{n-1}\right) f\left(x_{n-1}\right)+\beta_{n}\left(f\left(x_{n}\right)-f\left(x_{n-1}\right)\right) \\
+ & \left(\beta_{n-1}-\beta_{n}\right)\left(I-\lambda_{n-1} \mu_{n-1} F\right) G\left(J_{r_{n-1}} y_{n-1}\right) \\
+ & \left(1-\beta_{n}\right)\left[\left(I-\lambda_{n} \mu_{n} F\right) G\left(J_{r_{n}} y_{n}\right)\right. \\
& -\left(I-\lambda_{n} \mu_{n} F\right) G\left(J_{r_{n-1}} y_{n-1}\right) \\
& \left.+\left(\lambda_{n-1} \mu_{n-1}-\lambda_{n} \mu_{n}\right) F G\left(J_{r_{n-1}} y_{n-1}\right)\right] .
\end{aligned}
$$

It follows from Proposition 13(iii) and (109) that

$$
\begin{aligned}
& \left\|x_{n+1}-x_{n}\right\| \\
& \leq\left|\beta_{n}-\beta_{n-1}\right|\left\|f\left(x_{n-1}\right)\right\|+\beta_{n}\left\|f\left(x_{n}\right)-f\left(x_{n-1}\right)\right\| \\
& +\left|\beta_{n-1}-\beta_{n}\right|\left\|\left(I-\lambda_{n-1} \mu_{n-1} F\right) G\left(J_{r_{n-1}} y_{n-1}\right)\right\|+\left(1-\beta_{n}\right) \\
& \times\left[\left\|\left(I-\lambda_{n} \mu_{n} F\right) G\left(J_{r_{\mathrm{n}}} y_{n}\right)-\left(I-\lambda_{n} \mu_{n} F\right) G\left(J_{r_{n-1}} y_{n-1}\right)\right\|\right. \\
& \left.+\left|\lambda_{n-1} \mu_{n-1}-\lambda_{n} \mu_{n}\right|\left\|F G\left(J_{r_{n-1}} y_{n-1}\right)\right\|\right] \\
& \leq\left|\beta_{n}-\beta_{n-1}\right|\left\|f\left(x_{n-1}\right)\right\|+\beta_{n} \rho\left\|x_{n}-x_{n-1}\right\| \\
& +\left|\beta_{n-1}-\beta_{n}\right|\left\|\left(I-\lambda_{n-1} \mu_{n-1} F\right) G\left(J_{r_{n-1}} y_{n-1}\right)\right\| \\
& +\left(1-\beta_{n}\right)\left[\left(1-\lambda_{n} \mu_{n}\left(1-\sqrt{\frac{1-\delta}{\lambda}}\right)\right)\right. \\
& \times\left\|G\left(J_{r_{n}} y_{n}\right)-G\left(J_{r_{n-1}} y_{n-1}\right)\right\| \\
& \left.+\left|\lambda_{n} \mu_{n}-\lambda_{n-1} \mu_{n-1}\right|\left\|F G\left(J_{r_{n-1}} y_{n-1}\right)\right\|\right] \\
& \leq\left|\beta_{n}-\beta_{n-1}\right|\left\|f\left(x_{n-1}\right)\right\|+\beta_{n} \rho\left\|x_{n}-x_{n-1}\right\| \\
& +\left|\beta_{n-1}-\beta_{n}\right|\left\|\left(I-\lambda_{n-1} \mu_{n-1} F\right) G\left(J_{r_{n-1}} y_{n-1}\right)\right\| \\
& +\left(1-\beta_{n}\right)\left[\left(1-\lambda_{n} \mu_{n}\left(1-\sqrt{\frac{1-\delta}{\lambda}}\right)\right)\right. \\
& \times\left\|J_{r_{n}} y_{n}-J_{r_{n-1}} y_{n-1}\right\| \\
& \left.+\left|\lambda_{n} \mu_{n}-\lambda_{n-1} \mu_{n-1}\right|\left\|F G\left(J_{r_{n-1}} y_{n-1}\right)\right\|\right] \\
& \leq\left|\beta_{n}-\beta_{n-1}\right|\left\|f\left(x_{n-1}\right)\right\|+\beta_{n} \rho\left\|x_{n}-x_{n-1}\right\| \\
& +\left|\beta_{n-1}-\beta_{n}\right|\left\|\left(I-\lambda_{n-1} \mu_{n-1} F\right) G\left(J_{r_{n-1}} y_{n-1}\right)\right\| \\
& +\left(1-\beta_{n}\right)\left[\left(1-\lambda_{n} \mu_{n}\left(1-\sqrt{\frac{1-\delta}{\lambda}}\right)\right)\right. \\
& \times\left(\left\|y_{n-1}-y_{n}\right\|+\left|r_{n-1}-r_{n}\right| M_{0}\right) \\
& \left.+\left|\lambda_{n} \mu_{n}-\lambda_{n-1} \mu_{n-1}\right|\left\|F G\left(J_{r_{n-1}} y_{n-1}\right)\right\|\right] \\
& \leq \beta_{n} \rho\left\|x_{n}-x_{n-1}\right\|+\left|\beta_{n}-\beta_{n-1}\right| \\
& \times\left(\left\|f\left(x_{n-1}\right)\right\|+\left\|\left(I-\lambda_{n-1} \mu_{n-1} F\right) G\left(J_{r_{n-1}} y_{n-1}\right)\right\|\right) \\
& +\left(1-\beta_{n}\right)\left\|y_{n-1}-y_{n}\right\|+\left|r_{n-1}-r_{n}\right| M_{0} \\
& +\left|\lambda_{n} \mu_{n}-\lambda_{n-1} \mu_{n-1}\right|\left\|F G\left(J_{r_{n-1}} y_{n-1}\right)\right\|
\end{aligned}
$$




$$
\begin{aligned}
\leq & \beta_{n} \rho\left\|x_{n}-x_{n-1}\right\|+\left|\beta_{n}-\beta_{n-1}\right| M_{2} \\
& +\left(1-\beta_{n}\right)\left\|y_{n-1}-y_{n}\right\|+\left|r_{n-1}-r_{n}\right| M_{2} \\
& +\left|\lambda_{n} \mu_{n}-\lambda_{n-1} \mu_{n-1}\right| M_{2} \\
= & \beta_{n} \rho\left\|x_{n}-x_{n-1}\right\|+\left(1-\beta_{n}\right)\left\|y_{n}-y_{n-1}\right\| \\
& +M_{2}\left(\left|\beta_{n}-\beta_{n-1}\right|+\left|\lambda_{n} \mu_{n}-\lambda_{n-1} \mu_{n-1}\right|\right. \\
& \left.\quad+\left|r_{n}-r_{n-1}\right|\right),
\end{aligned}
$$

where $\sup _{n \geq 0}\left\{\left\|f\left(x_{n}\right)\right\|+\left\|\left(I-\lambda_{n} \mu_{n} F\right) G\left(J_{r_{n}} y_{n}\right)\right\|,\left\|F G\left(J_{r_{n}} y_{n}\right)\right\|\right.$, $\left.M_{0}, M_{1}\right\} \leq M_{2}$ for some $M_{2}>0$.

Substituting (110) for (113), we get

$$
\begin{aligned}
& \left\|x_{n+1}-x_{n}\right\| \\
& \leq \beta_{n} \rho\left\|x_{n}-x_{n-1}\right\|+\left(1-\beta_{n}\right)\left\|y_{n}-y_{n-1}\right\| \\
& \quad+M_{2}\left(\left|\beta_{n}-\beta_{n-1}\right|+\left|\lambda_{n} \mu_{n}-\lambda_{n-1} \mu_{n-1}\right|\right. \\
& \left.\quad \quad+\left|r_{n}-r_{n-1}\right|\right) \\
& \quad \beta_{n} \rho\left\|x_{n}-x_{n-1}\right\|+\left(1-\beta_{n}\right) \\
& \quad \times\left[\left\|x_{n}-x_{n-1}\right\|+M_{1}\left(\left|r_{n}-r_{n-1}\right|+\left|\alpha_{n}-\alpha_{n-1}\right|\right)\right] \\
& \quad+M_{2}\left(\left|\beta_{n}-\beta_{n-1}\right|+\left|\lambda_{n} \mu_{n}-\lambda_{n-1} \mu_{n-1}\right|+\left|r_{n}-r_{n-1}\right|\right) \\
& \leq \\
& \quad\left(1-(1-\rho) \beta_{n}\right)\left\|x_{n}-x_{n-1}\right\| \\
& \quad+2 M_{2}\left[\left|\alpha_{n}-\alpha_{n-1}\right|+\left|\beta_{n}-\beta_{n-1}\right|\right. \\
& \left.\quad \quad+\left|\lambda_{n}-\lambda_{n-1}\right|+\left|\mu_{n}-\mu_{n-1}\right|+\left|r_{n}-r_{n-1}\right|\right] .
\end{aligned}
$$

Since it follows from conditions (i) and (iv) that $\sum_{n=0}^{\infty}(1-$ $\rho) \beta_{n}=\infty$ and

$$
\begin{gathered}
\sum_{n=0}^{\infty} 2 M_{2}\left[\left|\alpha_{n}-\alpha_{n-1}\right|+\left|\beta_{n}-\beta_{n-1}\right|+\left|\lambda_{n}-\lambda_{n-1}\right|\right. \\
\left.+\left|\mu_{n}-\mu_{n-1}\right|+\left|r_{n}-r_{n-1}\right|\right]<\infty,
\end{gathered}
$$

Lemma 4 is applicable to (114) and we obtain

$$
\lim _{n \rightarrow \infty}\left\|x_{n+1}-x_{n}\right\|=0 .
$$

By condition (iii) and (95), we have

$$
\begin{aligned}
& \left\|y_{n}-x_{n}\right\| \\
& =\left(1-\alpha_{n}\right)\left\|G\left(J_{r_{n}} x_{n}\right)-x_{n}\right\| \\
& \leq(1-a)\left(\left\|G\left(J_{r_{n}} x_{n}\right)-G\left(J_{r_{n}} y_{n}\right)\right\|\right. \\
& \left.+\left\|G\left(J_{r_{n}} y_{n}\right)-x_{n+1}\right\|+\left\|x_{n+1}-x_{n}\right\|\right) \\
& \leq(1-a)\left(\| x_{n}-y_{n}\right)\|+\| G\left(J_{r_{n}} y_{n}\right)-x_{n+1} \| \\
& \left.+\left\|x_{n+1}-x_{n}\right\|\right),
\end{aligned}
$$

which implies that

$$
\left\|y_{n}-x_{n}\right\| \leq \frac{1-a}{a}\left(\left\|G\left(J_{r_{n}} y_{n}\right)-x_{n+1}\right\|+\left\|x_{n+1}-x_{n}\right\|\right) \text {. }
$$

This together with (99)-(100) implies that

$$
\lim _{n \rightarrow \infty}\left\|x_{n}-y_{n}\right\|=0 \text {. }
$$

So we obtain

$$
\begin{aligned}
\left\|x_{n}-G\left(J_{r_{n}} x_{n}\right)\right\| & \leq\left\|x_{n}-y_{n}\right\|+\left\|y_{n}-G\left(J_{r_{n}} x_{n}\right)\right\| \\
& \leq\left\|x_{n}-y_{n}\right\|+\alpha_{n}\left\|x_{n}-G\left(J_{r_{n}} x_{n}\right)\right\| \\
& \leq\left\|x_{n}-y_{n}\right\|+b\left\|x_{n}-G\left(J_{r_{n}} x_{n}\right)\right\|,
\end{aligned}
$$

which implies that

$$
\left\|x_{n}-G\left(J_{r_{n}} x_{n}\right)\right\| \leq \frac{1}{1-b}\left\|x_{n}-y_{n}\right\|,
$$

and hence

$$
\lim _{n \rightarrow \infty}\left\|x_{n}-G\left(J_{r_{n}} x_{n}\right)\right\|=0 .
$$

Next let us show that $\lim _{n \rightarrow \infty}\left\|x_{n}-J_{r_{n}} x_{n}\right\|=0$ and $\lim _{n \rightarrow \infty}\left\|x_{n}-G x_{n}\right\|=0$.

Indeed, for simplicity, put $v=\Pi_{C}\left(u-\mu_{2} B_{2} u\right), \widehat{x}_{n}=J_{r_{n}} x_{n}$, $u_{n}=\Pi_{C}\left(\hat{x}_{n}-\mu_{2} B_{2} \widehat{x}_{n}\right)$ and $v_{n}=\Pi_{C}\left(u_{n}-\mu_{1} B_{1} u_{n}\right)$. Then $u=$ $\Pi_{C}\left(v-\mu_{1} B_{1} v\right)$, and $v_{n}=G \hat{x}_{n}=G\left(J_{r_{n}} x_{n}\right)$ for all $n \geq 0$. It is clear from (95) that

$$
\begin{aligned}
\left\|y_{n}-u\right\|^{2} & \leq \alpha_{n}\left\|x_{n}-u\right\|^{2}+\left(1-\alpha_{n}\right)\left\|G\left(J_{r_{n}} x_{n}\right)-u\right\|^{2} \\
& =\alpha_{n}\left\|x_{n}-u\right\|^{2}+\left(1-\alpha_{n}\right)\left\|v_{n}-u\right\|^{2} .
\end{aligned}
$$

Utilizing Lemma 14, we have

$$
\begin{aligned}
\left\|u_{n}-v\right\|^{2} & =\left\|\Pi_{C}\left(\widehat{x}_{n}-\mu_{2} B_{2} \widehat{x}_{n}\right)-\Pi_{C}\left(u-\mu_{2} B_{2} u\right)\right\|^{2} \\
& \leq\left\|\widehat{x}_{n}-u-\mu_{2}\left(B_{2} \widehat{x}_{n}-B_{2} u\right)\right\|^{2} \\
& \leq\left\|\widehat{x}_{n}-u\right\|^{2}-2 \mu_{2}\left(\alpha_{2}-\kappa^{2} \mu_{2}\right)\left\|B_{2} \widehat{x}_{n}-B_{2} u\right\|^{2},
\end{aligned}
$$

$$
\begin{aligned}
\left\|v_{n}-u\right\|^{2} & =\left\|\Pi_{C}\left(u_{n}-\mu_{1} B_{1} u_{n}\right)-\Pi_{C}\left(v-\mu_{1} B_{1} v\right)\right\|^{2} \\
& \leq\left\|u_{n}-v-\mu_{1}\left(B_{1} u_{n}-B_{1} v\right)\right\|^{2} \\
& \leq\left\|u_{n}-v\right\|^{2}-2 \mu_{1}\left(\alpha_{1}-\kappa^{2} \mu_{1}\right)\left\|B_{1} u_{n}-B_{1} v\right\|^{2} .
\end{aligned}
$$

Substituting (124) for (125), we obtain

$$
\begin{aligned}
\left\|v_{n}-u\right\|^{2} \leq & \left\|\widehat{x}_{n}-u\right\|^{2}-2 \mu_{2}\left(\alpha_{2}-\kappa^{2} \mu_{2}\right)\left\|B_{2} \widehat{x}_{n}-B_{2} u\right\|^{2} \\
& -2 \mu_{1}\left(\alpha_{1}-\kappa^{2} \mu_{1}\right)\left\|B_{1} u_{n}-B_{1} v\right\|^{2} \\
\leq & \left\|x_{n}-u\right\|^{2}-2 \mu_{2}\left(\alpha_{2}-\kappa^{2} \mu_{2}\right)\left\|B_{2} \widehat{x}_{n}-B_{2} u\right\|^{2} \\
& -2 \mu_{1}\left(\alpha_{1}-\kappa^{2} \mu_{1}\right)\left\|B_{1} u_{n}-B_{1} v\right\|^{2},
\end{aligned}
$$


which, together with (123), implies that

$$
\begin{aligned}
& \left\|y_{n}-u\right\|^{2} \\
& \leq \alpha_{n}\left\|x_{n}-u\right\|^{2}+\left(1-\alpha_{n}\right)\left\|v_{n}-u\right\|^{2} \\
& \leq \alpha_{n}\left\|x_{n}-u\right\|^{2}+\left(1-\alpha_{n}\right) \\
& \quad \times\left[\left\|x_{n}-u\right\|^{2}-2 \mu_{2}\left(\alpha_{2}-\kappa^{2} \mu_{2}\right)\left\|B_{2} \widehat{x}_{n}-B_{2} u\right\|^{2}\right. \\
& \left.\quad-2 \mu_{1}\left(\alpha_{1}-\kappa^{2} \mu_{1}\right)\left\|B_{1} u_{n}-B_{1} v\right\|^{2}\right] \\
& =\left\|x_{n}-u\right\|^{2}-2\left(1-\alpha_{n}\right) \\
& \quad \times\left[\mu_{2}\left(\alpha_{2}-\kappa^{2} \mu_{2}\right)\left\|B_{2} \widehat{x}_{n}-B_{2} u\right\|^{2}\right. \\
& \left.\quad+\mu_{1}\left(\alpha_{1}-\kappa^{2} \mu_{1}\right)\left\|B_{1} u_{n}-B_{1} v\right\|^{2}\right] .
\end{aligned}
$$

It immediately follows that

$$
\begin{aligned}
& 2\left(1-\alpha_{n}\right)\left[\mu_{2}\left(\alpha_{2}-\kappa^{2} \mu_{2}\right)\left\|B_{2} \widehat{x}_{n}-B_{2} u\right\|^{2}\right. \\
& \left.\quad+\mu_{1}\left(\alpha_{1}-\kappa^{2} \mu_{1}\right)\left\|B_{1} u_{n}-B_{1} v\right\|^{2}\right] \\
& \leq\left\|x_{n}-u\right\|^{2}-\left\|y_{n}-u\right\|^{2} \\
& \leq\left(\left\|x_{n}-u\right\|+\left\|y_{n}-u\right\|\right)\left\|x_{n}-y_{n}\right\| .
\end{aligned}
$$

Since $\left\{x_{n}\right\}$ and $\left\{y_{n}\right\}$ are bounded and $0<\mu_{i}<\alpha_{i} / \kappa^{2}$ for $i=$ 1,2 , we deduce from (119) and condition (iii) that

$$
\begin{aligned}
& \lim _{n \rightarrow \infty}\left\|B_{2} \widehat{x}_{n}-B_{2} u\right\|=0, \\
& \lim _{n \rightarrow \infty}\left\|B_{1} u_{n}-B_{1} v\right\|=0 .
\end{aligned}
$$

Utilizing Proposition 3 and Lemma 6, we have

$$
\begin{aligned}
&\left\|u_{n}-v\right\|^{2} \\
&=\left\|\Pi_{C}\left(\widehat{x}_{n}-\mu_{2} B_{2} \widehat{x}_{n}\right)-\Pi_{C}\left(u-\mu_{2} B_{2} u\right)\right\|^{2} \\
& \leq\left\langle\widehat{x}_{n}-\mu_{2} B_{2} \widehat{x}_{n}-\left(u-\mu_{2} B_{2} u\right), J\left(u_{n}-v\right)\right\rangle \\
&=\left\langle\widehat{x}_{n}-u, J\left(u_{n}-v\right)\right\rangle+\mu_{2}\left\langle B_{2} p-B_{2} \widehat{x}_{n}, J\left(u_{n}-v\right)\right\rangle \\
& \leq \frac{1}{2}\left[\left\|\widehat{x}_{n}-u\right\|^{2}+\left\|u_{n}-v\right\|^{2}-g_{1}\left(\left\|\widehat{x}_{n}-u_{n}-(u-v)\right\|\right)\right] \\
&+\mu_{2}\left\|B_{2} u-B_{2} \widehat{x}_{n}\right\|\left\|u_{n}-v\right\|,
\end{aligned}
$$

which implies that

$$
\begin{gathered}
\left\|u_{n}-v\right\|^{2} \leq\left\|\widehat{x}_{n}-u\right\|^{2}-g_{1}\left(\left\|\widehat{x}_{n}-u_{n}-(u-v)\right\|\right) \\
+2 \mu_{2}\left\|B_{2} u-B_{2} \widehat{x}_{n}\right\|\left\|u_{n}-v\right\| .
\end{gathered}
$$

In the same way, we derive

$$
\begin{aligned}
&\left\|v_{n}-u\right\|^{2} \\
&=\left\|\Pi_{C}\left(u_{n}-\mu_{1} B_{1} u_{n}\right)-\Pi_{C}\left(v-\mu_{1} B_{1} v\right)\right\|^{2} \\
& \leq\left\langle u_{n}-\mu_{1} B_{1} u_{n}-\left(v-\mu_{1} B_{1} v\right), J\left(v_{n}-u\right)\right\rangle \\
&=\left\langle u_{n}-v, J\left(v_{n}-u\right)\right\rangle+\mu_{1}\left\langle B_{1} v-B_{1} u_{n}, J\left(v_{n}-u\right)\right\rangle \\
& \leq \frac{1}{2}\left[\left\|u_{n}-v\right\|^{2}+\left\|v_{n}-u\right\|^{2}-g_{2}\left(\left\|u_{n}-v_{n}+(u-v)\right\|\right)\right] \\
&+\mu_{1}\left\|B_{1} v-B_{1} u_{n}\right\|\left\|v_{n}-u\right\|,
\end{aligned}
$$

which implies that

$$
\begin{gathered}
\left\|v_{n}-u\right\|^{2} \leq\left\|u_{n}-v\right\|^{2}-g_{2}\left(\left\|u_{n}-v_{n}+(u-v)\right\|\right) \\
+2 \mu_{1}\left\|B_{1} v-B_{1} u_{n}\right\|\left\|v_{n}-u\right\| .
\end{gathered}
$$

Substituting (131) for (133), we get

$$
\begin{aligned}
\left\|v_{n}-u\right\|^{2} \leq & \left\|\widehat{x}_{n}-u\right\|^{2}-g_{1}\left(\left\|\widehat{x}_{n}-u_{n}-(u-v)\right\|\right) \\
& -g_{2}\left(\left\|u_{n}-v_{n}+(u-v)\right\|\right) \\
& +2 \mu_{2}\left\|B_{2} u-B_{2} \widehat{x}_{n}\right\|\left\|u_{n}-v\right\| \\
& +2 \mu_{1}\left\|B_{1} v-B_{1} u_{n}\right\|\left\|v_{n}-u\right\| \\
\leq & \left\|x_{n}-u\right\|^{2}-g_{1}\left(\left\|\widehat{x}_{n}-u_{n}-(u-v)\right\|\right) \\
& -g_{2}\left(\left\|u_{n}-v_{n}+(u-v)\right\|\right) \\
& +2 \mu_{2}\left\|B_{2} u-B_{2} \widehat{x}_{n}\right\|\left\|u_{n}-v\right\| \\
& +2 \mu_{1}\left\|B_{1} v-B_{1} u_{n}\right\|\left\|v_{n}-u\right\|,
\end{aligned}
$$

which, together with (123), implies that

$$
\begin{aligned}
& \left\|y_{n}-u\right\|^{2} \\
& \leq \alpha_{n}\left\|x_{n}-u\right\|^{2}+\left(1-\alpha_{n}\right)\left\|v_{n}-u\right\|^{2} \\
& \leq \alpha_{n}\left\|x_{n}-u\right\|^{2}+\left(1-\alpha_{n}\right) \\
& \times\left[\left\|x_{n}-u\right\|^{2}-g_{1}\left(\left\|\widehat{x}_{n}-u_{n}-(u-v)\right\|\right)\right. \\
& \quad-g_{2}\left(\left\|u_{n}-v_{n}+(u-v)\right\|\right) \\
& \quad+2 \mu_{2}\left\|B_{2} u-B_{2} \widehat{x}_{n}\right\|\left\|u_{n}-v\right\| \\
& \left.\quad+2 \mu_{1}\left\|B_{1} v-B_{1} u_{n}\right\|\left\|v_{n}-u\right\|\right] \\
& =\left\|x_{n}-u\right\|^{2}-\left(1-\alpha_{n}\right) \\
& \times\left[g_{1}\left(\left\|\widehat{x}_{n}-u_{n}-(u-v)\right\|\right)\right. \\
& \left.\quad+g_{2}\left(\left\|u_{n}-v_{n}+(u-v)\right\|\right)\right] \\
& +2\left(1-\alpha_{n}\right)\left(\mu_{2}\left\|B_{2} u-B_{2} \widehat{x}_{n}\right\|\left\|u_{n}-v\right\|\right. \\
& \left.\quad+\mu_{1}\left\|B_{1} v-B_{1} u_{n}\right\|\left\|v_{n}-u\right\|\right) .
\end{aligned}
$$


It immediately follows that

$$
\begin{aligned}
& \left(1-\alpha_{n}\right)\left[g_{1}\left(\left\|\widehat{x}_{n}-u_{n}-(u-v)\right\|\right)\right. \\
& \left.+g_{2}\left(\left\|u_{n}-v_{n}+(u-v)\right\|\right)\right] \\
& \leq\left\|x_{n}-u\right\|^{2}-\left\|y_{n}-u\right\|^{2} \\
& +2\left(1-\alpha_{n}\right)\left(\mu_{2}\left\|B_{2} u-B_{2} \widehat{x}_{n}\right\|\left\|u_{n}-v\right\|\right. \\
& \left.\quad+\mu_{1}\left\|B_{1} v-B_{1} u_{n}\right\|\left\|v_{n}-u\right\|\right) \\
& \leq\left(\left\|x_{n}-u\right\|+\left\|y_{n}-u\right\|\right)\left\|x_{n}-y_{n}\right\| \\
& +2 \mu_{2}\left\|B_{2} u-B_{2} \widehat{x}_{n}\right\|\left\|u_{n}-v\right\| \\
& +2 \mu_{1}\left\|B_{1} v-B_{1} u_{n}\right\|\left\|v_{n}-u\right\| .
\end{aligned}
$$

Since $\left\{x_{n}\right\},\left\{y_{n}\right\},\left\{u_{n}\right\}$, and $\left\{v_{n}\right\}$ are bounded, we deduce from (119), (129), and condition (iii) that

$$
\begin{aligned}
& \lim _{n \rightarrow \infty} g_{1}\left(\left\|\widehat{x}_{n}-u_{n}-(u-v)\right\|\right)=0, \\
& \lim _{n \rightarrow \infty} g_{2}\left(\left\|u_{n}-v_{n}+(u-v)\right\|\right)=0 .
\end{aligned}
$$

Utilizing the properties of $g_{1}$ and $g_{2}$, we get

$$
\begin{aligned}
& \lim _{n \rightarrow \infty}\left\|\widehat{x}_{n}-u_{n}-(u-v)\right\|=0, \\
& \lim _{n \rightarrow \infty}\left\|u_{n}-v_{n}+(u-v)\right\|=0,
\end{aligned}
$$

which hence yields

$$
\begin{aligned}
\left\|\widehat{x}_{n}-v_{n}\right\| \leq & \left\|\widehat{x}_{n}-u_{n}-(u-v)\right\| \\
& +\left\|u_{n}-v_{n}+(u-v)\right\| \longrightarrow 0 \quad \text { as } n \longrightarrow \infty .
\end{aligned}
$$

That is,

$$
\lim _{n \rightarrow \infty}\left\|J_{r_{n}} x_{n}-G\left(J_{r_{n}} x_{n}\right)\right\|=\lim _{n \rightarrow \infty}\left\|\widehat{x}_{n}-v_{n}\right\|=0 .
$$

Note that

$$
\left\|x_{n}-J_{r_{n}} x_{n}\right\| \leq\left\|x_{n}-G\left(J_{r_{n}} x_{n}\right)\right\|+\left\|G\left(J_{r_{n}} x_{n}\right)-J_{r_{n}} x_{n}\right\| .
$$

So from (122) and (140) we have

$$
\lim _{n \rightarrow \infty}\left\|x_{n}-J_{r_{n}} x_{n}\right\|=0
$$

which, together with (122), leads to

$$
\begin{aligned}
\left\|x_{n}-G x_{n}\right\| \leq & \left\|x_{n}-G\left(J_{r_{n}} x_{n}\right)\right\|+\left\|G\left(J_{r_{n}} x_{n}\right)-G x_{n}\right\| \\
\leq & \left\|x_{n}-G\left(J_{r_{n}} x_{n}\right)\right\| \\
& +\left\|J_{r_{n}} x_{n}-x_{n}\right\| \longrightarrow 0 \text { as } n \longrightarrow \infty .
\end{aligned}
$$

That is,

$$
\lim _{n \rightarrow \infty}\left\|x_{n}-G x_{n}\right\|=0
$$

In addition, utilizing Lemma 9 we obtain from $\left\{r_{n}\right\} \subset[\varepsilon, \infty)$ that

$$
\left\|x_{n}-J_{\varepsilon} x_{n}\right\| \leq 2\left\|x_{n}-J_{r_{n}} x_{n}\right\|,
$$

which, together with (142), implies that

$$
\lim _{n \rightarrow \infty}\left\|x_{n}-J_{\varepsilon} x_{n}\right\|=0
$$

\section{Define a mapping}

$$
W x=(1-\theta) J_{\varepsilon} x+\theta G x, \quad \forall x \in C,
$$

where $\theta$ is a constant in $(0,1)$. Then by Lemma 10 , we know that $\operatorname{Fix}(W)=\operatorname{Fix}\left(J_{\varepsilon}\right) \cap \operatorname{Fix}(G)=\Delta$. We observe that

$$
\begin{aligned}
\left\|x_{n}-W x_{n}\right\| & =\left\|(1-\theta)\left(x_{n}-J_{\varepsilon} x_{n}\right)+\theta\left(x_{n}-G x_{n}\right)\right\| \\
& \leq(1-\theta)\left\|x_{n}-J_{\varepsilon} x_{n}\right\|+\theta\left\|x_{n}-G x_{n}\right\| .
\end{aligned}
$$

So from (144) and (146) we obtain

$$
\lim _{n \rightarrow \infty}\left\|x_{n}-W x_{n}\right\|=0 .
$$

Now, we claim that

$$
\limsup _{n \rightarrow \infty}\left\langle f(p)-p, J\left(x_{n}-p\right)\right\rangle \leq 0,
$$

where $p=s-\lim _{t \rightarrow 0} x_{t}$, with $x_{t}$ being the fixed point of the contraction $x \mapsto t f(x)+(1-t) W x$ of $C$ into itself (due to Lemma 12). Then $x_{t}$ solves the fixed point equation $x_{t}=t f\left(x_{t}\right)+(1-t) W x_{t}$. Thus we have

$$
\left\|x_{t}-x_{n}\right\|=\left\|(1-t)\left(W x_{t}-x_{n}\right)+t\left(f\left(x_{t}\right)-x_{n}\right)\right\| .
$$

By Lemma 5 we conclude that

$$
\begin{aligned}
\left\|x_{t}-x_{n}\right\|^{2}= & \left\|(1-t)\left(W x_{t}-x_{n}\right)+t\left(f\left(x_{t}\right)-x_{n}\right)\right\|^{2} \\
\leq & (1-t)^{2}\left\|W x_{t}-x_{n}\right\|^{2} \\
& +2 t\left\langle f\left(x_{t}\right)-x_{n}, J\left(x_{t}-x_{n}\right)\right\rangle \\
\leq & (1-t)^{2}\left(\left\|W x_{t}-W x_{n}\right\|+\left\|W x_{n}-x_{n}\right\|\right)^{2} \\
& +2 t\left\langle f\left(x_{t}\right)-x_{n}, J\left(x_{t}-x_{n}\right)\right\rangle \\
\leq & (1-t)^{2}\left(\left\|x_{t}-x_{n}\right\|+\left\|W x_{n}-x_{n}\right\|\right)^{2} \\
& +2 t\left\langle f\left(x_{t}\right)-x_{n}, J\left(x_{t}-x_{n}\right)\right\rangle \\
= & (1-t)^{2}\left[\left\|x_{t}-x_{n}\right\|^{2}+2\left\|x_{t}-x_{n}\right\|\left\|W x_{n}-x_{n}\right\|\right. \\
& \left.\quad+\left\|W x_{n}-x_{n}\right\|^{2}\right] \\
& +2 t\left\langle f\left(x_{t}\right)-x_{t}, J\left(x_{t}-x_{n}\right)\right\rangle \\
& +2 t\left\langle x_{t}-x_{n}, J\left(x_{t}-x_{n}\right)\right\rangle \\
= & \left(1-2 t+t^{2}\right)\left\|x_{t}-x_{n}\right\|^{2}+f_{n}(t) \\
& +2 t\left\langle f\left(x_{t}\right)-x_{t}, J\left(x_{t}-x_{n}\right)\right\rangle+2 t\left\|x_{t}-x_{n}\right\|^{2},
\end{aligned}
$$


where

$$
\begin{aligned}
f_{n}(t)= & (1-t)^{2}\left(2\left\|x_{t}-x_{n}\right\|+\left\|x_{n}-W x_{n}\right\|\right) \\
& \times\left\|x_{n}-W x_{n}\right\| \longrightarrow 0, \quad \text { as } n \longrightarrow \infty .
\end{aligned}
$$

It follows from (152) that

$$
\left\langle x_{t}-f\left(x_{t}\right), J\left(x_{t}-x_{n}\right)\right\rangle \leq \frac{t}{2}\left\|x_{t}-x_{n}\right\|^{2}+\frac{1}{2 t} f_{n}(t) .
$$

Letting $n \rightarrow \infty$ in (154) and noticing (153), we derive

$$
\limsup _{n \rightarrow \infty}\left\langle x_{t}-f\left(x_{t}\right), J\left(x_{t}-x_{n}\right)\right\rangle \leq \frac{t}{2} M_{3},
$$

where $M_{3}>0$ is a constant such that $\left\|x_{t}-x_{n}\right\|^{2} \leq M_{3}$ for all $t \in(0,1)$ and $n \geq 0$. Taking $t \rightarrow 0$ in (155), we have

$$
\limsup _{t \rightarrow 0} \limsup _{n \rightarrow \infty}\left\langle x_{t}-f\left(x_{t}\right), J\left(x_{t}-x_{n}\right)\right\rangle \leq 0 .
$$

On the other hand, we have

$$
\begin{aligned}
\left\langle f(p)-p, J\left(x_{n}-p\right)\right\rangle \\
=\left\langle f(p)-p, J\left(x_{n}-p\right)\right\rangle-\left\langle f(p)-p, J\left(x_{n}-x_{t}\right)\right\rangle \\
\quad+\left\langle f(p)-p, J\left(x_{n}-x_{t}\right)\right\rangle-\left\langle f(p)-x_{t}, J\left(x_{n}-x_{t}\right)\right\rangle \\
\quad+\left\langle f(p)-x_{t}, J\left(x_{n}-x_{t}\right)\right\rangle-\left\langle f\left(x_{t}\right)-x_{t}, J\left(x_{n}-x_{t}\right)\right\rangle \\
\quad+\left\langle f\left(x_{t}\right)-x_{t}, J\left(x_{n}-x_{t}\right)\right\rangle \\
=\left\langle f(p)-p, J\left(x_{n}-p\right)-J\left(x_{n}-x_{t}\right)\right\rangle \\
\quad+\left\langle x_{t}-p, J\left(x_{n}-x_{t}\right)\right\rangle+\left\langle f(p)-f\left(x_{t}\right), J\left(x_{n}-x_{t}\right)\right\rangle \\
\quad+\left\langle f\left(x_{t}\right)-x_{t}, J\left(x_{n}-x_{t}\right)\right\rangle .
\end{aligned}
$$

It follows that

$$
\begin{aligned}
\limsup _{n \rightarrow \infty}\left\langle f(p)-p, J\left(x_{n}-p\right)\right\rangle \\
\leq \limsup _{n \rightarrow \infty}\left\langle f(p)-p, J\left(x_{n}-p\right)-J\left(x_{n}-x_{t}\right)\right\rangle \\
\quad+\left\|x_{t}-p\right\| \limsup _{n \rightarrow \infty}\left\|x_{n}-x_{t}\right\| \\
\quad+\rho\left\|p-x_{t}\right\| \limsup _{n \rightarrow \infty}\left\|x_{n}-x_{t}\right\| \\
\quad+\limsup _{n \rightarrow \infty}\left\langle f\left(x_{t}\right)-x_{t}, J\left(x_{n}-x_{t}\right)\right\rangle .
\end{aligned}
$$

Taking into account that $x_{t} \rightarrow p$ as $t \rightarrow 0$, we have from (156)

$$
\begin{aligned}
& \limsup _{n \rightarrow \infty}\left\langle f(p)-p, J\left(x_{n}-p\right)\right\rangle \\
& \quad=\limsup _{t \rightarrow 0} \limsup _{n \rightarrow \infty}\left\langle f(p)-p, J\left(x_{n}-p\right)\right\rangle \\
& \quad \leq \limsup \limsup _{n \rightarrow \infty}\left\langle f(p)-p, J\left(x_{n}-p\right)-J\left(x_{n}-x_{t}\right)\right\rangle .
\end{aligned}
$$

Since $X$ has a uniformly Frechet differentiable norm, the duality mapping $J$ is norm-to-norm uniformly continuous on bounded subsets of $X$. Consequently, the two limits are interchangeable and hence (150) holds. It is clear from (150) that

$$
\limsup _{n \rightarrow \infty}\left\langle f(p)-p, J\left(x_{n+1}-p\right)\right\rangle \leq 0
$$

Finally, let us show that $x_{n} \rightarrow p$ as $n \rightarrow \infty$. We observe that

$$
\begin{aligned}
\left\|y_{n}-p\right\|^{2} & \leq \alpha_{n}\left\|x_{n}-p\right\|^{2}+\left(1-\alpha_{n}\right)\left\|G\left(J_{r_{n}} x_{n}\right)-p\right\|^{2} \\
& \leq \alpha_{n}\left\|x_{n}-p\right\|^{2}+\left(1-\alpha_{n}\right)\left\|x_{n}-p\right\|^{2} \\
& =\left\|x_{n}-p\right\|^{2},
\end{aligned}
$$

and hence

$$
\begin{aligned}
& \left\|x_{n+1}-p\right\|^{2} \\
& =\| \beta_{n}\left(f\left(x_{n}\right)-f(p)\right) \\
& +\left(1-\beta_{n}\right)\left[G\left(J_{r_{n}} y_{n}\right)-\lambda_{n} \mu_{n} F G\left(J_{r_{n}} y_{n}\right)-p\right] \\
& +\beta_{n}(f(p)-p) \|^{2} \\
& \leq \| \beta_{n}\left(f\left(x_{n}\right)-f(p)\right) \\
& +\left(1-\beta_{n}\right)\left[G\left(J_{r_{n}} y_{n}\right)-\lambda_{n} \mu_{n} F G\left(J_{r_{n}} y_{n}\right)-p\right] \|^{2} \\
& +2 \beta_{n}\left\langle f(p)-p, J\left(x_{n+1}-p\right)\right\rangle \\
& \leq \beta_{n}\left\|f\left(x_{n}\right)-f(p)\right\|^{2} \\
& +\left(1-\beta_{n}\right)\left\|G\left(J_{r_{n}} y_{n}\right)-\lambda_{n} \mu_{n} F G\left(J_{r_{n}} y_{n}\right)-p\right\|^{2} \\
& +2 \beta_{n}\left\langle f(p)-p, J\left(x_{n+1}-p\right)\right\rangle \\
& =\beta_{n}\left\|f\left(x_{n}\right)-f(p)\right\|^{2} \\
& +\left(1-\beta_{n}\right) \|\left(I-\lambda_{n} \mu_{n} F\right) G\left(J_{r_{n}} y_{n}\right) \\
& -\left(I-\lambda_{n} \mu_{n} F\right) p-\lambda_{n} \mu_{n} F p \|^{2} \\
& +2 \beta_{n}\left\langle f(p)-p, J\left(x_{n+1}-p\right)\right\rangle \\
& \leq \beta_{n} \rho\left\|x_{n}-p\right\|^{2} \\
& +\left(1-\beta_{n}\right)\left[\left\|\left(I-\lambda_{n} \mu_{n} F\right) G\left(J_{r_{n}} y_{n}\right)-\left(I-\lambda_{n} \mu_{n} F\right) p\right\|\right. \\
& \left.+\lambda_{n} \mu_{n}\|F p\|\right]^{2} \\
& +2 \beta_{n}\left\langle f(p)-p, J\left(x_{n+1}-p\right)\right\rangle
\end{aligned}
$$




$$
\begin{aligned}
& \leq \beta_{n} \rho\left\|x_{n}-p\right\|^{2}+\left(1-\beta_{n}\right) \\
& \times\left[\left(1-\lambda_{n} \mu_{n}\left(1-\sqrt{\frac{1-\delta}{\lambda}}\right)\right)\left\|G\left(J_{r_{n}} y_{n}\right)-p\right\|\right. \\
& \left.+\lambda_{n} \mu_{n}\|F p\|\right]^{2} \\
& +2 \beta_{n}\left\langle f(p)-p, J\left(x_{n+1}-p\right)\right\rangle \\
& \leq \beta_{n} \rho\left\|x_{n}-p\right\|^{2} \\
& +\left(1-\beta_{n}\right)\left(\left\|y_{n}-p\right\|+\lambda_{n} \mu_{n}\|F p\|\right)^{2} \\
& +2 \beta_{n}\left\langle f(p)-p, J\left(x_{n+1}-p\right)\right\rangle \\
& \leq \beta_{n} \rho\left\|x_{n}-p\right\|^{2} \\
& +\left(1-\beta_{n}\right)\left(\left\|x_{n}-p\right\|+\lambda_{n} \mu_{n}\|F p\|\right)^{2} \\
& +2 \beta_{n}\left\langle f(p)-p, J\left(x_{n+1}-p\right)\right\rangle \\
& =\beta_{n} \rho\left\|x_{n}-p\right\|^{2} \\
& +\left(1-\beta_{n}\right)\left[\left\|x_{n}-p\right\|^{2}+\lambda_{n} \mu_{n}\|F p\|\right. \\
& \left.\times\left(2\left\|x_{n}-p\right\|+\lambda_{n} \mu_{n}\|F p\|\right)\right] \\
& +2 \beta_{n}\left\langle f(p)-p, J\left(x_{n+1}-p\right)\right\rangle \\
& \leq\left(1-(1-\rho) \beta_{n}\right)\left\|x_{n}-p\right\|^{2} \\
& +\lambda_{n} \mu_{n}\|F p\|\left(2\left\|x_{n}-p\right\|+\lambda_{n} \mu_{n}\|F p\|\right) \\
& +2 \beta_{n}\left\langle f(p)-p, J\left(x_{n+1}-p\right)\right\rangle \\
& =\left(1-(1-\rho) \beta_{n}\right)\left\|x_{n}-p\right\|^{2} \\
& +(1-\rho) \beta_{n}\left\{\frac{\lambda_{n} \mu_{n}}{\beta_{n}} \frac{\|F p\|\left(2\left\|x_{n}-p\right\|+\lambda_{n} \mu_{n}\|F p\|\right)}{1-\rho}\right. \\
& \left.+\frac{2\left\langle f(p)-p, J\left(x_{n+1}-p\right)\right\rangle}{1-\rho}\right\} .
\end{aligned}
$$

Taking into account (160) and conditions (i), (ii), we obtain that $\sum_{n=0}^{\infty}(1-\rho) \beta_{n}=\infty$ and

$$
\begin{aligned}
\limsup _{n \rightarrow \infty}\left\{\frac{\lambda_{n} \mu_{n}}{\beta_{n}} \frac{\|F p\|\left(2\left\|x_{n}-p\right\|+\lambda_{n} \mu_{n}\|F p\|\right)}{1-\rho}\right. \\
\left.+\frac{2\left\langle f(p)-p, J\left(x_{n+1}-p\right)\right\rangle}{1-\rho}\right\} \leq 0 .
\end{aligned}
$$

Therefore, applying Lemma 4 to (162), we infer that

$$
\lim _{n \rightarrow \infty}\left\|x_{n}-p\right\|=0
$$

This completes the proof.
Remark 19. As pointed out in [12, Remark 3.2], the sequences $\left\{\lambda_{n}\right\},\left\{\mu_{n}\right\}$, and $\left\{\beta_{n}\right\}$ can be taken, which satisfy the conditions in Theorem 18. As a matter of fact, put $\lambda_{n}=(1+n)^{-5 / 6}, \mu_{n}=$ 1 , and $\beta_{n}=(1+n)^{-2 / 3}$ for all $n \geq 0$. Then there hold the following statements:

(i) $\lim _{n \rightarrow \infty} \beta_{n}=0$ and $\sum_{n=0}^{\infty} \beta_{n}=\infty$,

(ii) $\lim _{n \rightarrow \infty}\left(\lambda_{n} \mu_{n}\right) / \beta_{n}=0$,

(iii) $\sum_{n=0}^{\infty}\left|\beta_{n+1}-\beta_{n}\right|<\infty, \sum_{n=0}^{\infty}\left|\lambda_{n+1}-\lambda_{n}\right|<\infty$, and $\sum_{n=0}^{\infty}\left|\mu_{n+1}-\mu_{n}\right|<\infty$.

By the careful analysis of the proof of Theorem 18, we can obtain the following result. Because its proof is much simpler than that of Theorem 18, we omit its proof.

Theorem 20. Let $X$ be a uniformly convex and 2-uniformly smooth Banach space and let $A$ be an $m$-accretive operator in $X$ such that $C=\overline{D(A)}$ is convex. Let $\Pi_{C}$ be a sunny nonexpansive retraction from $X$ onto $C$. Let the mapping $B_{i}: C \rightarrow X$ be $\alpha_{i-}$ inverse strongly accretive for $i=1,2$, let $f: X \rightarrow C$ be a contractive map with coefficient $\rho \in(0,1)$, and let $F: X \rightarrow$ $X$ be $\delta$-strongly accretive and $\lambda$-strictly pseudocontractive with $\delta+\lambda>1$. Assume that $\Delta=A^{-1}(0) \cap \Omega \neq \emptyset$, where $\Omega$ is the fixed point set of the mapping $G=\Pi_{C}\left(I-\mu_{1} B_{1}\right) \Pi_{C}\left(I-\mu_{2} B_{2}\right)$ with $0<\mu_{i}<\alpha_{i} / \kappa^{2}$ for $i=1,2$. Given sequences $\left\{\lambda_{n}\right\}_{n=0}^{\infty}$ in $[0,1],\left\{\alpha_{n}\right\}_{n=0}^{\infty},\left\{\beta_{n}\right\}_{n=0}^{\infty}$ in $(0,1]$, and $\left\{r_{n}\right\}_{n=0}^{\infty}$ in $[\varepsilon, \infty)$ for some $\varepsilon>0$, suppose that there hold the following conditions:

(i) $\lim _{n \rightarrow \infty} \beta_{n}=0$ and $\sum_{n=0}^{\infty} \beta_{n}=\infty$,

(ii) $\lim _{n \rightarrow \infty} \lambda_{n} / \beta_{n}=0$ and $\sum_{n=0}^{\infty}\left|\lambda_{n+1}-\lambda_{n}\right|<\infty$,

(iii) $\left\{\alpha_{n}\right\} \subset[a, b]$ for some $a, b \in(0,1)$,

(iv) $\sum_{n=0}^{\infty}\left|\alpha_{n+1}-\alpha_{n}\right|<\infty, \sum_{n=0}^{\infty}\left|\beta_{n+1}-\beta_{n}\right|<\infty$, and Then for any given point $x_{0} \in X$, the sequence $\left\{x_{n}\right\}$ generated by

$$
\begin{aligned}
& y_{n}=\alpha_{n} x_{n}+\left(1-\alpha_{n}\right) G\left(J_{r_{n}} x_{n}\right), \\
& x_{n+1}=\beta_{n} f\left(x_{n}\right)+\left(1-\beta_{n}\right)\left[y_{n}-\lambda_{n} F\left(y_{n}\right)\right], \\
& \forall n \geq 0,
\end{aligned}
$$

converges strongly to $p \in \Delta$, which is a unique solution of the VIP (44).

Remark 21. Our Theorems 16-20 improve, extend, supplement, and develop Cai and $\mathrm{Bu}$ [13, Theorem 3.1] and Ceng et al. [12, Theorems 3.1-3.3] in the following aspects.

(i) The problem of finding a point $p \in A^{-1}(0) \cap \Omega$ in our Theorems $16-20$ is very different from everyone of both the problem of finding a point $p \in \bigcap_{n} \operatorname{Fix}\left(S_{n}\right) \cap \Omega$ in Cai and $\mathrm{Bu}[13$, Theorem 3.1] and the problem of finding a point $p \in A^{-1}(0)$ in Ceng et al. [12, Theorems 3.1-3.3]. There is no doubt that our problem of finding a point $p \in A^{-1}(0) \cap \Omega$ is more general than the problem of finding a point $p \in A^{-1}(0)$ in [12, Theorems 3.1-3.3]. 
(ii) Compared with the choice of iterative parameters in [12, Theorems 3.1-3.3], the choice of iterative parameters in our Theorems $16-20$ is the same as that in [12, Theorems 3.1-3.3].

(iii) The iterative schemes in [12, Theorems 3.1-3.3] are extended to develop the iterative schemes in our Theorems $16-20$ by virtue of the iterative scheme of [13, Theorems 3.1]. The iterative schemes in our Theorems 16-20 are more advantageous and more flexible than the iterative schemes of [12, Theorems 3.1-3.3] because they involve solving two problems: the GSVI (16) and the problem of finding zeros of an $m$-accretive operator.

(iv) The iterative schemes in our Theorems 16-20 are very different from everyone in both [13, Theorem 3.1] and [12, Theorems 3.1-3.3] because the iterative scheme in our Theorem 16 is implicit and because the mapping $G$ in [13, Theorem 3.1] and the mapping $J_{r_{n}}$ in [12, Theorems 3.1-3.3] are replaced by the same composite mapping $G \circ J_{r_{n}}$ in the iterative schemes of our Theorems $16-20$.

(v) Cai and Bu's proof in [13, Theorem 3.1] depends on the argument techniques in [16], the inequality in 2uniformly smooth Banach spaces (see Lemma 2), and the inequality in smooth and uniform convex Banach spaces (see Proposition 3). Because the composite mapping $G \circ J_{r_{n}}$ appears in the iterative schemes in our Theorems 16-20, the proof of our Theorems 1620 depends on the argument techniques in [16], the inequality in 2-uniformly smooth Banach spaces (see Lemma 2), the inequality in smooth and uniform convex Banach spaces (see Proposition 3), and the properties of the resolvent of an $m$-accretive operator (see Lemmas 8 and 9), the Banach limit (see Lemma 11) and the strongly accretive and strictly pseudocontractive mapping (see Proposition 13).

\section{Acknowledgments}

This research was partially supported by the National Science Foundation of China (11071169), Innovation Program of Shanghai Municipal Education Commission (09ZZ133), and Ph.D. Program Foundation of Ministry of Education of China (20123127110002). This research was partially supported by a Grant from NSC 102-2115-M-037-001.

\section{References}

[1] F. E. Browder, "Convergence theorems for sequences of nonlinear operators in Banach spaces," Mathematische Zeitschrift, vol. 100, pp. 201-225, 1967.

[2] L. C. Zeng, G. M. Lee, and N. C. Wong, "Ishikawa iteration with errors for approximating fixed points of strictly pseudocontractive mappings of Browder-Petryshyn type," Taiwanese Journal of Mathematics, vol. 10, no. 1, pp. 87-99, 2006.

[3] H. Iiduka and W. Takahashi, "Strong convergence theorems for nonexpansive mappings and inverse-strongly monotone mappings," Nonlinear Analysis. Theory, Methods \& Applications A, vol. 61, no. 3, pp. 341-350, 2005.

[4] W. Takahashi and M. Toyoda, "Weak convergence theorems for nonexpansive mappings and monotone mappings," Journal of Optimization Theory and Applications, vol. 118, no. 2, pp. 417428, 2003.

[5] H. Iiduka, W. Takahashi, and M. Toyoda, "Approximation of solutions of variational inequalities for monotone mappings," Panamerican Mathematical Journal, vol. 14, no. 2, pp. 49-61, 2004.

[6] Y. Takahashi, K. Hashimoto, and M. Kato, "On sharp uniform convexity, smoothness, and strong type, cotype inequalities," Journal of Nonlinear and Convex Analysis, vol. 3, no. 2, pp. 267281, 2002.

[7] R. Chen and Z. Zhu, "Viscosity approximation method for accretive operator in Banach space," Nonlinear Analysis. Theory, Methods \& Applications A, vol. 69, no. 4, pp. 1356-1363, 2008.

[8] L.-C. Ceng, A. R. Khan, Q. H. Ansari, and J.-C. Yao, "Strong convergence of composite iterative schemes for zeros of $m$ accretive operators in Banach spaces," Nonlinear Analysis. Theory, Methods \& Applications A, vol. 70, no. 5, pp. 1830-1840, 2009.

[9] L. C. Zeng, N. C. Wong, and J. C. Yao, "Convergence of hybrid steepest-descent methods for generalized variational inequalities," Acta Mathematica Sinica, vol. 22, no. 1, pp. 1-12, 2006.

[10] L. C. Zeng, N. C. Wong, and J. C. Yao, "Convergence analysis of modified hybrid steepest-descent methods with variable parameters for variational inequalities," Journal of Optimization Theory and Applications, vol. 132, no. 1, pp. 51-69, 2007.

[11] L.-C. Ceng, Q. H. Ansari, and J.-C. Yao, "Mann-type steepestdescent and modified hybrid steepest-descent methods for variational inequalities in Banach spaces," Numerical Functional Analysis and Optimization, vol. 29, no. 9-10, pp. 987-1033, 2008.

[12] L.-C. Ceng, Q. H. Ansari, S. Schaible, and J.-C. Yao, "Hybrid viscosity approximation method for zeros of $m$-accretive operators in Banach spaces," Numerical Functional Analysis and Optimization, vol. 33, no. 2, pp. 142-165, 2012.

[13] G. Cai and S. Bu, "Convergence analysis for variational inequality problems and fixed point problems in 2-uniformly smooth and uniformly convex Banach spaces," Mathematical and Computer Modelling, vol. 55, no. 3-4, pp. 538-546, 2012.

[14] R. U. Verma, "On a new system of nonlinear variational inequalities and associated iterative algorithms," Mathematical Sciences Research Hot-Line, vol. 3, no. 8, pp. 65-68, 1999.

[15] J.-L. Lions and G. Stampacchia, "Variational inequalities," Communications on Pure and Applied Mathematics, vol. 20, pp. 493519, 1967.

[16] L.-C. Ceng, C.-Y. Wang, and J.-C. Yao, "Strong convergence theorems by a relaxed extragradient method for a general system of variational inequalities," Mathematical Methods of Operations Research, vol. 67, no. 3, pp. 375-390, 2008.

[17] G. M. Korpelevič, "An extragradient method for finding saddle points and for other problems," Ėkonomika i Matematicheskie Metody, vol. 12, no. 4, pp. 747-756, 1976.

[18] F. Facchinei and J.-S. Pang, Finite-dimensional variational inequalities and complementarity problems, vol. 1-2, Springer, New York, NY, USA, 2003.

[19] A. N. Iusem and B. F. Svaiter, "A variant of Korpelevich's method for variational inequalities with a new search strategy," Optimization, vol. 42, no. 4, pp. 309-321, 1997. 
[20] L.-C. Ceng and J.-C. Yao, "An extragradient-like approximation method for variational inequality problems and fixed point problems," Applied Mathematics and Computation, vol. 190, no. 1, pp. 205-215, 2007.

[21] Y. Censor, A. Gibali, and S. Reich, "Two extensions of Korpelevich's extragradient method for solving the variational inequality problem in Euclidean space," Tech. Rep., 2010.

[22] L.-C. Zeng and J.-C. Yao, "Strong convergence theorem by an extragradient method for fixed point problems and variational inequality problems," Taiwanese Journal of Mathematics, vol. 10, no. 5, pp. 1293-1303, 2006.

[23] N. Nadezhkina and W. Takahashi, "Weak convergence theorem by an extragradient method for nonexpansive mappings and monotone mappings," Journal of Optimization Theory and Applications, vol. 128, no. 1, pp. 191-201, 2006.

[24] L.-C. Ceng, Q. H. Ansari, and J.-C. Yao, "An extragradient method for solving split feasibility and fixed point problems," Computers \& Mathematics with Applications, vol. 64, no. 4, pp. 633-642, 2012.

[25] L. C. Ceng, M. Teboulle, and J. C. Yao, "Weak convergence of an iterative method for pseudomonotone variational inequalities and fixed-point problems," Journal of Optimization Theory and Applications, vol. 146, no. 1, pp. 19-31, 2010.

[26] L.-C. Ceng, Q. H. Ansari, and J.-C. Yao, "Relaxed extragradient methods for finding minimum-norm solutions of the split feasibility problem," Nonlinear Analysis. Theory, Methods \& Applications A, vol. 75, no. 4, pp. 2116-2125, 2012.

[27] L.-C. Ceng, Q. H. Ansari, and J.-C. Yao, "Relaxed extragradient iterative methods for variational inequalities," Applied Mathematics and Computation, vol. 218, no. 3, pp. 1112-1123, 2011.

[28] R. U. Verma, "Projection methods, algorithms, and a new system of nonlinear variational inequalities," Computers \& Mathematics with Applications, vol. 41, no. 7-8, pp. 1025-1031, 2001.

[29] L.-C. Ceng, Q. H. Ansari, N.-C. Wong, and J.-C. Yao, "An extragradient-like approximation method for variational inequalities and fixed point problems," Fixed Point Theory and Applications, vol. 2011, article 22, 18 pages, 2011.

[30] L.-C. Ceng, N. Hadjisavvas, and N.-C. Wong, "Strong convergence theorem by a hybrid extragradient-like approximation method for variational inequalities and fixed point problems," Journal of Global Optimization, vol. 46, no. 4, pp. 635-646, 2010.

[31] L.-C. Ceng, S.-M. Guu, and J.-C. Yao, "Finding common solutions of a variational inequality, a general system of variational inequalities, and a fixed-point problem via a hybrid extragradient method," Fixed Point Theory and Applications, vol. 2011, Article ID 626159, 22 pages, 2011.

[32] Y. Yao, Y.-C. Liou, S. M. Kang, and Y. Yu, "Algorithms with strong convergence for a system of nonlinear variational inequalities in Banach spaces," Nonlinear Analysis. Theory, Methods \& Applications A, vol. 74, no. 17, pp. 6024-6034, 2011.

[33] K. Aoyama, H. Iiduka, and W. Takahashi, "Weak convergence of an iterative sequence for accretive operators in Banach spaces," Fixed Point Theory and Applications, vol. 2004, Article ID 35390, 13 pages, 2006.

[34] H.-K. Xu, "Viscosity approximation methods for nonexpansive mappings," Journal of Mathematical Analysis and Applications, vol. 298, no. 1, pp. 279-291, 2004.

[35] L.-C. Ceng, H.-K. Xu, and J.-C. Yao, "Strong convergence of an iterative method with perturbed mappings for nonexpansive and accretive operators," Numerical Functional Analysis and Optimization, vol. 29, no. 3-4, pp. 324-345, 2008.
[36] H. K. Xu, "Inequalities in Banach spaces with applications," Nonlinear Analysis. Theory, Methods \& Applications A, vol. 16, no. 12, pp. 1127-1138, 1991.

[37] S. Kamimura and W. Takahashi, "Strong convergence of a proximal-type algorithm in a Banach space," SIAM Journal on Optimization, vol. 13, no. 3, pp. 938-945, 2002.

[38] H. K. Xu and T. H. Kim, "Convergence of hybrid steepestdescent methods for variational inequalities," Journal of Optimization Theory and Applications, vol. 119, no. 1, pp. 185-201, 2003.

[39] S. Reich, "Weak convergence theorems for nonexpansive mappings in Banach spaces," Journal of Mathematical Analysis and Applications, vol. 67, no. 2, pp. 274-276, 1979.

[40] V. Barbu, Nonlinear Semigroups and Differential Equations in Banach Spaces, Noordhoff, Leiden, The Netherlands, 1976.

[41] H.-K. Xu, "Strong convergence of an iterative method for nonexpansive and accretive operators," Journal of Mathematical Analysis and Applications, vol. 314, no. 2, pp. 631-643, 2006.

[42] R. E. Bruck, Jr., "Properties of fixed-point sets of nonexpansive mappings in Banach spaces," Transactions of the American Mathematical Society, vol. 179, pp. 251-262, 1973.

[43] W. Takahashi, Nonlinear functional analysis, Fixed Point Theory and Its Applications, Yokohama, Yokohama, Japan, 2000, (Japanese). 


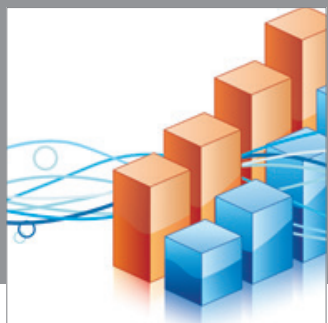

Advances in

Operations Research

mansans

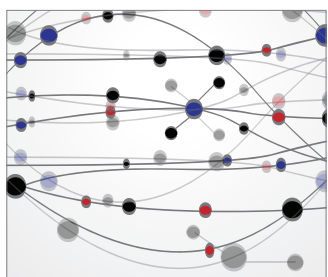

The Scientific World Journal
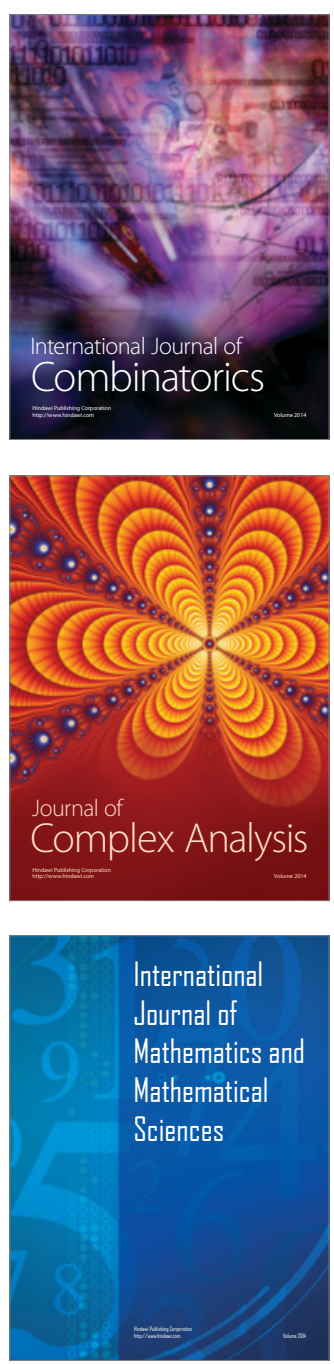
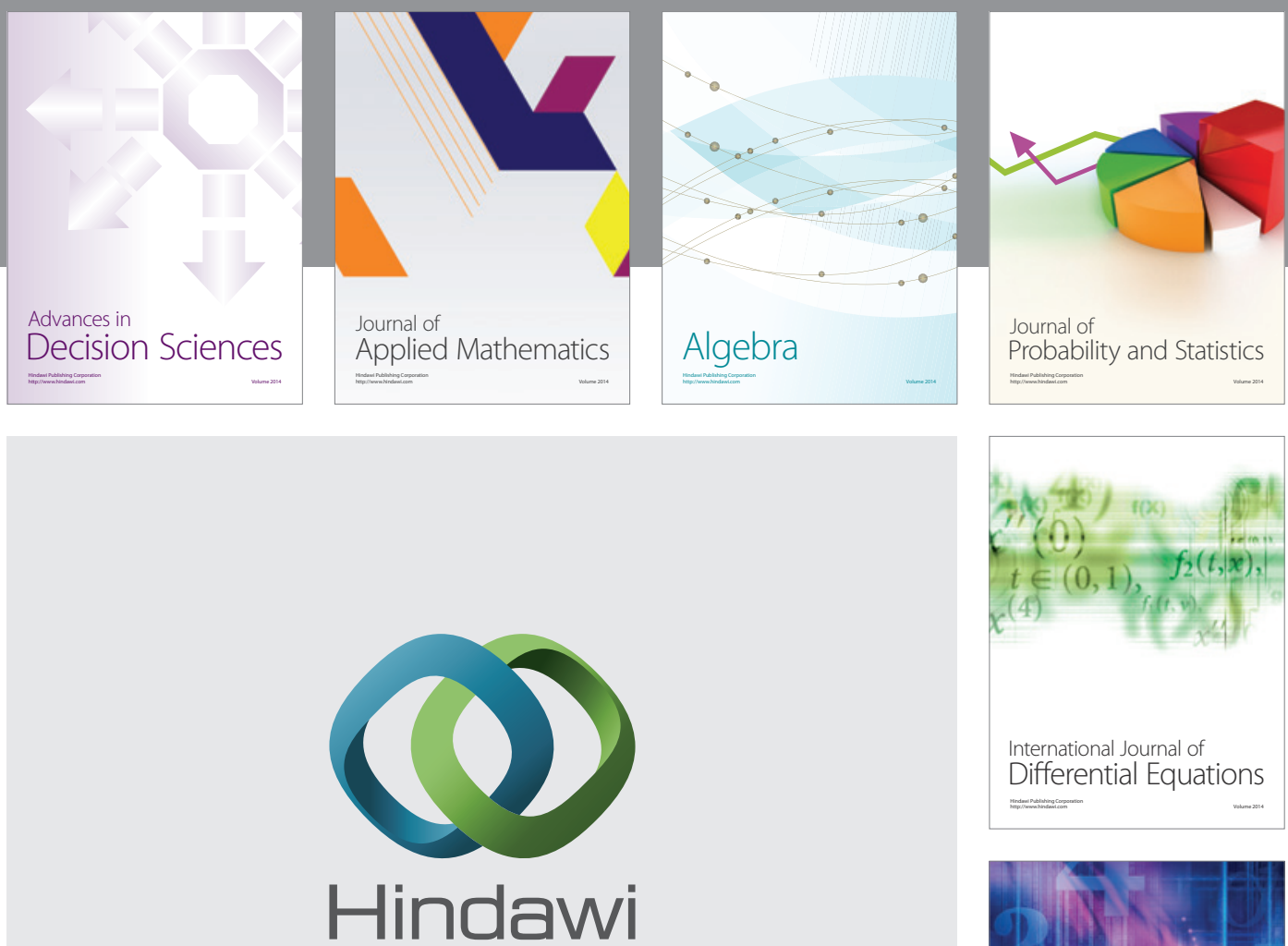

Submit your manuscripts at http://www.hindawi.com
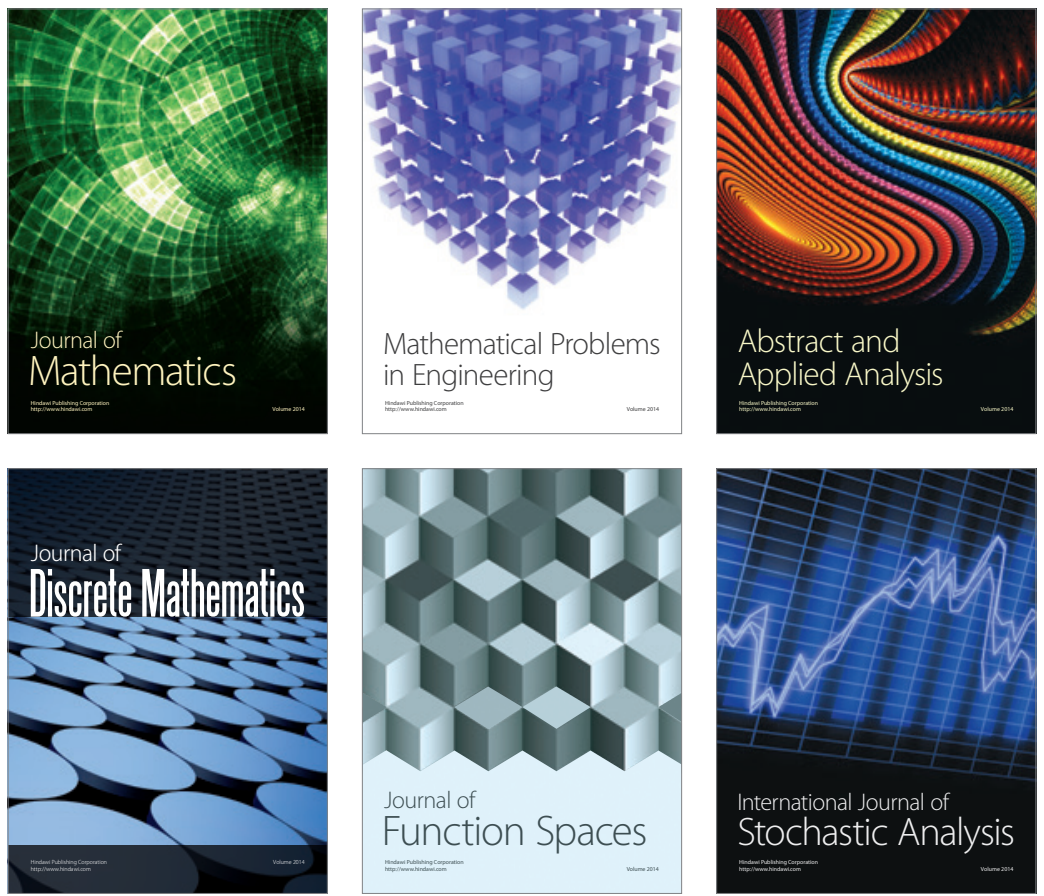

Journal of

Function Spaces

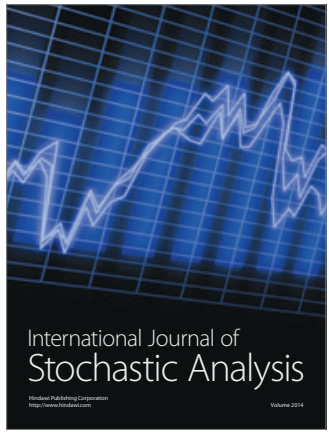

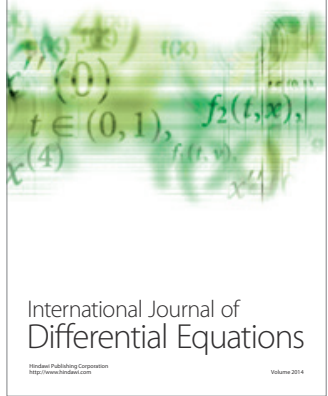
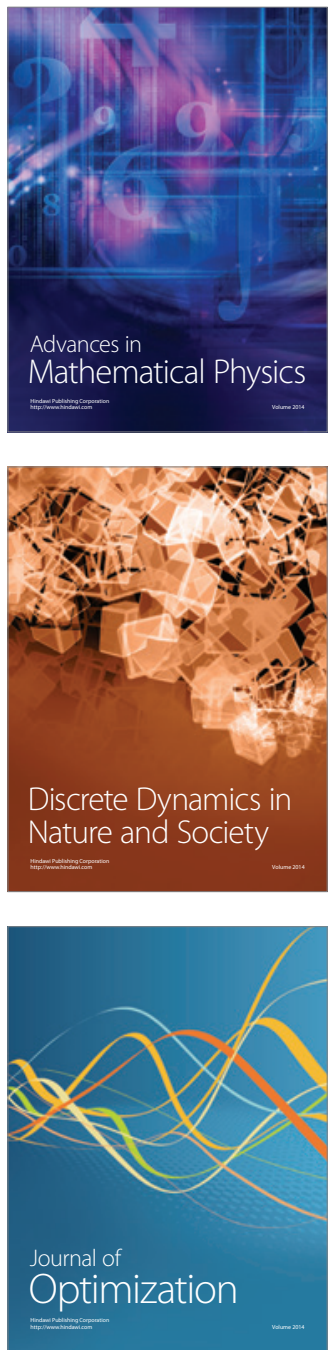